\title{
Psychosocial interventions for survivors of rape and sexual assault experienced during adulthood (Protocol)
}

Brown SJ, Khasteganan N, Brown K, Hegarty K, Carter GJ, Tarzia L, Feder G, O'Doherty L

Brown SJ, Khasteganan N, Brown K, Hegarty K, Carter GJ, Tarzia L, Feder G, O'Doherty L.

Psychosocial interventions for survivors of rape and sexual assault experienced during adulthood.

Cochrane Database of Systematic Reviews 2019, Issue 11. Art. No.: CD013456.

DOI: 10.1002/14651858.CD013456.

www.cochranelibrary.com

Psychosocial interventions for survivors of rape and sexual assault experienced during adulthood (Protocol) Copyright $\odot 2019$ The Cochrane Collaboration. Published by John Wiley \& Sons, Ltd. 
TABLE OF CONTENTS

HEADER 1

ABSTRACT

BACKGROUND

OBJECTIVES

METHODS

ACKNOWLEDGEMENTS

REFERENCES

APPENDICES

CONTRIBUTIONS OF AUTHORS

DECLARATIONS OF INTEREST

SOURCES OF SUPPORT 
[Intervention Protocol]

\section{Psychosocial interventions for survivors of rape and sexual assault experienced during adulthood}

Sarah J Brown ${ }^{1,2}$, Nazanin Khasteganan ${ }^{1}$, Katherine Brown ${ }^{1}$, Kelsey Hegarty3,4, Grace J Carter ${ }^{1}$, Laura Tarzia3,4, Gene Feder ${ }^{5}$, Lorna O'Doherty 1,3

1 Faculty of Health and Life Sciences, Coventry University, Coventry, UK. ${ }^{2}$ School of Law and Criminology, University of the Sunshine Coast, Sippy Downs, Australia. ${ }^{3}$ Department of General Practice, The University of Melbourne, Melbourne, Australia. ${ }^{4}$ The Royal Women's Hospital, Victoria, Australia. ${ }^{5}$ Centre for Academic Primary Care, Population Health Sciences, Bristol Medical School, University of Bristol, Bristol, UK

Contact address: Sarah J Brown, Faculty of Health and Life Sciences, Coventry University, Priory Street, Coventry, CV1 5FB, UK. Sarah.Brown@Coventry.ac.uk, sbrown2@usc.edu.au.

Editorial group: Cochrane Developmental, Psychosocial and Learning Problems Group

Publication status and date: New, published in Issue 11, 2019.

Citation: Brown SJ, Khasteganan N, Brown K, Hegarty K, Carter GJ, Tarzia L, Feder G, O'Doherty L. Psychosocial interventions for survivors of rape and sexual assault experienced during adulthood. Cochrane Database of Systematic Reviews 2019, Issue 11. Art. No.: CD013456. DOI: 10.1002/14651858.CD013456.

Copyright ( 2019 The Cochrane Collaboration. Published by John Wiley \& Sons, Ltd.

\section{A B S T R A C T}

This is a protocol for a Cochrane Review (Intervention). The objectives are as follows:

To assess the effects of psychosocial interventions on mental health and well-being for survivors of rape and sexual assault experienced during adulthood. 


\section{B A C K G R O U N D}

\section{Description of the condition}

Rape and sexual assault are serious crimes, with the two terms being used to differentiate between different types of behaviours (offences). Sexual assault is an act of physical, psychological and emotional violation in the form of a sexual act, inflicted on someone without their consent. It can involve forcing or manipulating someone to witness or participate in sexual acts. An attempt to engage a person in such activities is referred to as attempted sexual assault. Legislation varies both between and within countries in respect of the activities that meet the criteria for sexual assault. For example, in the UK, sexual assault is based on 'touching' without consent, while the Supreme Court of Canada held that the act of sexual assault does not depend solely on contact with any specific part of the human anatomy but rather the act of a sexual nature that violates the sexual integrity of the victim. Rape is a specific form of sexual assault, defined by the World Health Organisation (WHO 2002) as "physically forced or otherwise coerced penetration - even if slight - of the vulva or anus, using a penis, other body parts or an object. The attempt to do so is known as attempted rape." (quote; $p$ 149) There are differences in the types of acts that meet different legislative criteria for rape around the world (e.g. in some countries, rape only applies when a man commits the act against a female), but generally and broadly, the offence involves sexual penetration without consent. When children are raped or sexually assaulted, this is typically referred to as child sexual abuse (CSA), despite the fact that legislation for these offences may include the terms rape and sexual assault (e.g. rape of a minor, sexual assault of a child).

Rape and sexual assault are significantly under-reported; for example, only $23 \%$ of the 323,450 rapes or sexual assaults against individuals aged 12 years or older reported to the USA National Crime Victimization Survey in 2016 had been reported to the police (Morgan 2017), and just $17 \%$ of sexual assaults experienced since 16 years of age in the British Crime Survey in 2013/14 (ONS 2015). Thus, it is difficult to understand the full extent of the problem, with estimates varying widely depending on the definitions used and method of data collection. There are more population-based survey data available to estimate rape and sexual assault perpetrated by intimate partners, compared to non-partners (WHO/PAHO 2012). The lifetime prevalence of sexual violence perpetrated by an intimate partner reported by women aged 15 to 49 years in the WHO multi-country study ranged from $6 \%$ in Japan to $59 \%$ in Ethiopia (WHO 2005). In this study, 0.3 to $12 \%$ of women reported having been forced, after the age of 15 years, to have sexual intercourse or to perform a sexual act by someone other than an intimate partner. Estimates of prevalence using reports of perpetrators are rare. A cross-sectional survey of a randomly selected sample of men in South Africa revealed that $14.3 \%$ reported having raped their current or former wife or girlfriend, while one in five reported raping a woman who was not a partner (i.e. a stranger, acquaintance or family member) (Jewkes 2011).

Rape and sexual assault disproportionately affect women (Walby 2016). Research into men's experiences of rape and sexual assault has been characterised by small samples sizes and varying definitions, and thus, the prevalence of rape and sexual assault perpetrated against men is largely unknown. Social and legal marginalisation, exacerbated by gender-defined services, stigma and discrimination, all mean that the experiences of rape and sexual assault experienced by transgender people are hidden and poorly un- derstood (e.g. see Wirtz 2018). In relation to sexual identities, the 2010 National Intimate Partner and Sexual Violence Survey showed that one in five bisexual women were raped by a partner (relative to one in 10 heterosexual women); rates of sexual violence were also higher for gay men and bisexual men, compared to heterosexual men (Walters 2013).

There is growing application of syndemic (concurrent or sequential diseases that additively increase negative health consequences) frameworks to understand the way in which different exposures or conditions (e.g. intimate partner violence (IPV) and substance misuse) co-occur and exacerbate each other, producing new health problems such as HIV (Brennan 2012; Singer 2003). In the context of sexual violence, the approach highlights how structural factors, such as poverty and immigration status, and social aspects, such as different identities, disability, history of exploitation or sex work and lack of support systems, interact to produce health inequities and reinforce the disease burden (Willen 2017). The same factors reduce the capacity of research to bear witness to the experiences of those affected by constellations of social, political and economic factors. The limited evidence we do have on the hidden experiences of men, and other groups both silenced and at high risk, suggest trauma-related sequelae are similar across all groups (Coxell 2010).

Sexual assault is a serious public health and human rights problem (WHO 2013a). It has devastating effects on adult and child victims, their families, and communities. There are extensive immediate and long-term physical and mental health consequences for survivors. The consequences for adult and child victims include injuries, substance misuse, eating disorders, post-traumatic stress disorder (PTSD), anxiety, depression, self-harm and suicidality (WHO 2013a). Sexual and reproductive health problems represent the largest and most persistent physical health differences between women with and without exposure to rape or sexual assault, or both. Problems include unwanted pregnancy, sexually transmitted infections (WHO 2013a), urinary tract infections, painful sex, chronic pelvic pain and vaginal bleeding (Campbell 2002). For male victims, physical health consequences include genital and rectal injuries and erectile dysfunction (Tewkesbury 2007).

The mental health burden is substantial and similar across male and female victims (Coxell 2010; Tewkesbury 2007; Walker 2005; WHO 2013a). Sexual assault was ranked among the top three most traumatic life events in the US National Epidemiologic study (n $=34,653$; Pietrzak 2011). Participants in that study with a psychiatric diagnosis of PTSD were four times more likely to report exposure to sexual assault than controls, and $13 \%$ of women with PTSD had lifetime experience of sexual assault. PTSD is a psychiatric disorder that can follow exposure to psychological trauma and is associated with intrusive memories, nightmares, avoidance, and problems with sleep and concentration (Lerman 2019). These findings are consistent with the World Mental Health Survey (Liu 2017). Guina and colleagues reported no difference in PTSD symptoms and severity among men and women who had experienced sexual trauma (Guina 2016). Other mental health consequences include alcohol use disorders, eating disorders, anxiety, depression, selfharm and suicidality (WHO 2013a). Indirect pathways to poor longterm health outcomes are also of concern; for example, taking lifetime PTSD as a proxy, PTSD is associated with increased risk of hypertension, cardiovascular disease and gastrointestinal problems (Pietrzak 2011). Thus, the immense medical and psychological impacts of sexual violence exposure can lead to long-term disability. 
The negative effects of rape and sexual assault ripple across generations, having social and economic costs in addition to impacts on physical and mental health by affecting, for example, individuals' capacities to work and to participate in family and community life. Rape and sexual assault produce a significant social and economic burden, with lost productivity and police and criminal justice costs, in addition to the health and mental health burden. In the UK, each adult rape has been estimated to cost over $£ 73,000$ from psychological damage to a person, the physical impacts of associated injuries and illnesses, health service use, and economic losses (Home Office 2005). In the USA, the Centers for Disease Control and Prevention estimated that the lifetime cost of rape was US\$122,461 per victim, which amounted to a population economic burden of almost US $\$ 3.1$ trillion (Peterson 2017). This figure relates to data showing that over 25 million adults had been raped and included medical costs (39\%), lost work productivity relating to both victims and perpetrators (52\%), criminal justice costs (8\%), and other expenses such as victim property loss or damage (1\%). There are additional impacts to consider, from lost economic output to increased use of social services, impacts on family, capacity to parent, intergenerational transmission of trauma and violence, and effects for the wider community. Thus, providing accessible, evidence-based interventions in response to victims is not only a moral imperative, but an essential requirement to limit the consequences of rape and sexual assault across the lifespan and disrupt the costly pathways to poor health.

\section{Description of the intervention}

While there is a great deal of consensus that sexual assault and rape are highly detrimental to mental health, the conceptualisation of that harm has been the subject of debate (Campbell 2009). Early sexual assault interventions arose from a crisis theory orientation (e.g. Burgess 1974), which informed rape advocacy organisations (Koss 1987a). However, there is a lack of evidence for this approach and indications that some women with chronic symptoms needed more intensive treatment (Kilpatrick 1983). Cognitive-behavioural interventions that built on evidence-based anxiety treatments were adapted for this population in the 1970s, which included Stress Inoculation Training (SIT; Veronen 1983). Later, Prolonged Exposure Therapy (PET; Foa 1986) and Cognitive Processing Therapy (CPT; Resnick 1977) were developed and evaluated (see Vickerman 2009 for a review). Then, behavioural therapies, such as Eye Movement Desensitisation Reprocessing (EMDR; Shapiro 1995), received increased research attention and began to be evaluated in this population (e.g. Rothbaum 1997). These approaches sit within a trauma-response theoretical model (Goodman 1993; Herman 1992); however, the clinical diagnosis of PTSD risks pathologising victims (Berg 2002; Gilfus 1999), has been identified as re-traumatising and unhelpful by survivors, and perpetuates ethnocultural biases (Marsella 1996; Wasco 2003). Rape and sexual assault do not occur in social and cultural isolation (Campbell 2009). As highlighted recently by the \#MeToo movement, victims have to negotiate post-assault responses and help-seeking in hostile and doubting environments. This is due to a pervasive culture that propagates messages that victims are to blame, that they caused the assault or rape and deserved it (Buchwald 1993; Burt 1998; Lonsway 1994; Sandy 1998), if they are believed at all. Hence, violence against women scholars have advocated for an ecologically-informed trauma model of rape recovery (Koss 1991; Neville 1999), which takes these issues into account and highlights the different systems within which responses and support are provided, and moreover stress- es the importance of social as well as psychological responses. According to Kelly's ecological theory (Kelly 1966; Kelly 1968; Kelly 1971), individuals' and community organisations' responses are interdependent, resulting in each person having differential patterns of experiences depending on their ecological circumstances. Koss 1991 and Harvey 1996 adapted these ideas in their ecological model of rape recovery, which Campbell and colleagues used to evaluate legal, medical, and mental health systems' responses to survivors' needs and the influences on survivors' psychological, physical, and sexual health outcomes (Campbell 1998; Campbell 1999; Campbell 2001; Campbell 2004). The World Health Organization (WHO; Jewkes 2002; Krug 2002) and Center for Disease Control and Prevention (CDC 2004) have adapted this approach in the prevention of gender-based violence. This means that a wide range of interventions has been developed to support or respond (or both) to victims of sexual assault and rape. These include supportive therapies, whereby counsellors and/or specific sexual assault/rape support workers, advocates or advisors give support, information and advice to survivors. They may listen to victims and help them talk over their feelings and problems (BluePages 2012). Counsellors may offer debriefing, which allows emotional processing or ventilation by encouraging recollection, ventilation and reworking of the traumatic event (Rose 2002).

Psychosocial interventions "are interpersonal or informational activities, techniques, or strategies that target biological, behavioral, cognitive, emotional, interpersonal, social, or environmental factors with the aim of improving health functioning and well-being" (IOM 2015, p 5). They vary considerably as interventions target different combinations of these factors. For example, Sikkema and colleagues describe the development of a psychosocial intervention for South African women with sexual trauma histories (Sikkema 2018). The intervention included both individual and group sessions with psycho-education and focused on the following treatment themes: synergistic stress of sexual trauma and HIV; impact of trauma on health behaviours; safety, intimacy, power, and selfesteem; stressor identification and appraisal; adaptive versus maladaptive coping; social support; and reduction of shame and stigma. Group education sessions (e.g. Dognin 2017) and brief videobased interventions that provide psycho-education and modelling of coping strategies to survivors at the time of a sexual assault nurse examination (Miller 2015) have also been developed for this population. Sexual Assault Referral Centres (e.g. NHS 2015; Vandenberghe 2018) provide a range of initial response and support services, including in the UK, independent sexual violence advisors (ISVAs) who are non-psychologists trained to look after survivors' needs (Home Office 2017). This role was commissioned by Baroness Stern through the Home Office Violent Crime Unit in 2005. An ISVA is trained to ensure survivors receive care and understanding. Guidance sets out the core principles of an ISVA, which are to: tailor support to the individual's needs; provide accurate and impartial information; provide emotional and practical support to meet the survivor's needs; provide support before, during and after court; act as a single point of contact; ensure the safety of survivors and their dependants; and provide a professional service (Home Office 2017). Discussing the rape or sexual assault prior to court proceedings is seen as prejudicial to a trial (CPS 2002) and most psychological therapies include such discussion. For this reason, psychosocial interventions that are tailored to avoid such discussion can be a vital source of support to rape and sexual assault victims in the pre-trial period. Although many psychosocial interventions have demonstrated effectiveness, the findings have not been well syn- 
thesised, and it can be difficult to know what treatments are effective (IOM 2015).

Women have been the focus of interventions and services for rape and sexual assault; male, transgender and gender non-conforming/non-binary populations experience significant barriers in respect of accessing such interventions. This is also reflected in the evaluation literature, with services specifically for non-female populations receiving little or no evaluation and samples in evaluations of interventions being predominately female. Furthermore, the preponderance of white/Caucasian women attending services and participating across studies of interventions to date, further underscores the importance of undertaking this review; many subgroups remain hidden (e.g. men and boys) or highly vulnerable to abuse (migrant people, minority ethnic people, LGBT (lesbian, gay, bisexual and transgender) people, those involved in sexual exploitation and sex work), or both, and this is reflected in both practice and research contexts. This review has the potential to draw together experiences across studies among individuals typically under-represented in research, who share certain social, gender, ethnic and economic characteristics, to determine if the approaches under investigation respond differently for subgroups of survivors.

For the purposes of this review, we will include a wide range of psychosocial interventions (for definitions, see the list of psychological therapies on the Cochrane Common Mental Disorders (CCMD) website (cmd.cochrane.org/psychological-therapies-topics-list)). These include: (a) formal Cognitive Behavioural Therapy (CBT) and Trauma-Focused CBT (TF-CBT), and CBT-based techniques; (b) integrative therapies including SIT, PET, CPT; (c) behaviour therapies, such as EMDR and relaxation techniques, many of which are based on cognitive-behavioural processes (Freeman 2005); (d) third-wave CBT such as Acceptance and Commitment Therapy and mindfulness; (e) humanistic therapies such as supportive and non-directive therapy; ( $f$ ) other psychologically-orientated interventions such as art therapy; meditation; and narrative therapy; and (g) psychosocial interventions such as support and services delivered by mentors, support workers, advisors, or advocates (for example, independent sexual assault advisors (ISVAs), in the UK), and support groups.

Cognitive-behavioural processes can also be subclassified into three major classes (Dobson 2009): (1) cognitive re-structuring, which focuses on internal underlying beliefs and thoughts with the aim of challenging maladaptive thought patterns; (2) coping skills therapy, which targets the identification and alteration of cognitions and behaviours that may increase the impact of negative external events; and (3) problem-solving therapies, which combine cognitive re-structuring and coping skills therapy to change internal thought patterns and optimise responses to external negative events. Each of these three classes have a slightly different target for change, demonstrating the wide range of psychological interventions based upon cognitive-behavioural principles (Dobson 2009).

\section{See Appendix 1.}

\section{Why it is important to do this review}

Clinical and policy guidelines inform responses to rape and sexual assault (e.g. NICE 2018; WHO 2013b), but gaps remain in our knowledge of the most effective ways of intervening to improve health outcomes and prevent further victimisation. While there is moder- ate evidence on the consequences of sexual trauma (Description of the condition), it is less clear what happens to people's health and well-being over time, including in response to different interventions. Although post-traumatic stress is strongly associated with rape and sexual assault (e.g. Liu 2017), and there are theoretical understandings on the importance of early community response to mitigate it, the effectiveness of interventions in promoting survivor well-being is unclear. There is good evidence for the effects of psychological treatments in reducing mental health issues in children who have experienced sexual trauma (Gillies 2016), with CBT for sexually abused children with symptoms of post-traumatic stress showing the best evidence for reduction in mental health conditions (MacDonald 2012; MacMillan 2009). However, these conclusions cannot be extrapolated to adults who have experienced sexual trauma, and there has been no recent systematic review or metaanalysis examining the effects of intervention on this population.

Relative to IPV, sexual violence has received less attention in the research literature, and several prior or ongoing reviews focus on psychological interventions for IPV (Arroyo 2017; Tan 2018; Trabold 2018). While there is some overlap in the populations of interest, in that many sexual assaults and rapes occur within IPV, rape and sexual assault is not exclusive to IPV and there is a larger group of individuals who experience sexual trauma as adults who require support or interventions. Those reviews that have looked at rape and sexual assault have tended to focus on women (Parcesepe 2015) and children (Gillies 2016; MacDonald 2012), indicating that the experiences of men and transgender survivors are less represented in the literature. Similarly, the representation of sexual minorities and ethnic minorities is typically minimal in intervention studies, with studies rarely sufficiently powered to detect benefits and costs for specific user groups or subgroups of survivors. By pooling subgroups from different studies, the current review will have the potential to address some of the gaps on what works for whom, and under what circumstances. Other reviews have focused on diagnosis or outcome (i.e. PTSD) (Roberts 2015), psychological therapies (Bisson 2013) or combined pharmacotherapy and psychological therapies (Hetrick 2010) for PTSD, rather than the population/exposure (rape and sexual assault survivors). For most of the reviews, sexual assault and rape victims or survivors are children or adolescents or are a subset of the population. While these reviews are helpful in understanding appropriate therapies to combat PTSD specifically, not all sexual assault or rape victims experience PTSD, and the impacts of sexual trauma are broader than PTSD. Campbell and colleagues published a review in 2009 (Campbell 2009) and Regehr and colleagues a systematic review in 2013 (Regehr 2013) on interventions to reduce distress in adult victims of sexual assault and rape. These reviews are relevant; however, they are now 10 and six years out of date, respectively, and there have been developments in terms of interventions since their publication. The proposed review will examine the broader range of impacts of sexual trauma for all victims who experience rape and sexual assault as adults. Each of the previous two reviews included six studies and we identified 10 eligible studies in our scoping review. Hence, this review is feasible and addresses an important gap in the current literature.

\section{O B J E C T IVES}

To assess the effects of psychosocial interventions on mental health and well-being for survivors of rape and sexual assault experienced during adulthood. 


\section{METHODS}

\section{Criteria for considering studies for this review}

\section{Types of studies}

Any study that allocated individuals or clusters of individuals by a random or quasi-random method (whereby the method of allocation was not truly random such as alternate allocation, allocation by birth date, day shift etc.) to a psychosocial intervention for adult victims of rape or sexual assault compared with no intervention, usual care, waiting list, or minimal or active comparison (see 'Comparator intervention' under Types of interventions).

Studies will be eligible for inclusion in the review if they used random assignment to treatment and comparison groups or employed one of the following designs: quasi-randomised controlled trial (RCT) (non-randomised experimental design trials); cluster-RCT (instead of individual randomised trials, groups will be randomised) or cross-over trial (longitudinal studies where the participant receives a sequence of different treatments).

\section{Types of participants}

All adults aged 18 years and older, of any gender, who have experienced rape or sexual assault as an adult (i.e. aged 18 years and older), irrespective of a mental health diagnosis. Types of sexual assault will include rape, attempted rape, forced oral sex, anal sex, penetration with objects, touching of intimate parts and any sexual contact where consent was not given, as well as forcing or manipulating someone to witness sexual acts. We will include studies of participants who screened positive for exposure to sexual violence, even if they do not report what those behaviours were. We will include studies involving subsets of eligible participants provided that the subset includes at least $50 \%$ of those randomised and can be analysed separately. We will include studies of participants recruited in any setting (e.g. community, forensic, criminal justice, and health).

We will exclude samples made up entirely of individuals (adult or child) who were victims of rape, sexual assault, or sexual abuse during their childhood (aged 17 years and under), as well as samples of children (i.e. those younger than 18 years of age).

\section{Types of interventions}

\section{Experimental intervention}

The experimental intervention consists of any type of psychosocial and psychological intervention that targets recovery from sexual assault or rape, including the following.

1. Formal CBT, TF-CBT and CBT-based techniques.

2. Integrative therapies, including Stress Inoculation Training (SIT; Veronen 1983), Prolonged Exposure Therapy (PET; Foa 1986) and Cognitive Processing Therapy (CPT).

3. Behaviour therapies such as EMDR and relaxation techniques.

4. Third wave CBT (e.g. Acceptance and Commitment Therapy, mindfulness).

5. Humanistic therapies (e.g. supportive and non-directive therapy).

6. Other psychologically-orientated interventions (e.g. art therapy, meditation, trauma-informed body-based practices (e.g. embodied relational therapy, yoga and Tai Chi), narrative therapy).

7. Other psychosocial interventions, including support services delivered by mentors, support workers, advisors or advocates such as ISVAs in the UK, support groups, and coping interventions.

We will include interventions of any duration or frequency of treatment so long as the treatment meets the criteria stated above.

For all interventions, mode of intervention delivery will include one or more of the following: face-to-face; telephone; or computer-based delivery. We will include both individual and group delivery of the intervention.

\section{Comparator intervention}

Comparator interventions will consist of inactive controls, such as usual care, no treatment, delayed provision of psychological interventions (or waiting-list conditions), or pharmacological treatment only, and minimal interventions such as information provision. However, we will not exclude studies on the grounds that an active control group has been used (e.g. where an intervention from one category (CBT) is compared to an intervention from another category (psychosocial intervention), or different intensities or dosages of an intervention are compared). We recognise that there will be instances where researchers employ an active comparison condition for pragmatic or ethical reasons (e.g. the importance of offering some care or treatment to a survivor and that research studies may replicate this when designing or delivering an evaluation). In our analyses, we will strive to pool studies that conduct similar types of comparisons (i.e. active versus inactive or active versus active).

\section{Types of outcome measures}

We will not select studies based on the nature of the outcomes assessed. The review is designed to measure the effects of psychological therapies and psychosocial interventions for survivors of rape and sexual assault experienced during adulthood, based on a wide range of indicators of a person's health and well-being, particularly mental health and well-being. We are also mindful about evaluating harm and adverse consequences from therapies and other interventions.

\section{Primary outcomes}

1. Treatment efficacy, PTSD symptoms: response to treatment, determined by differences in scores for PTSD symptoms, assessed by independent observer or self-report. Validated observer-rated instruments include the Clinician-Administered PTSD Symptom Scale (Kulka 1988), Clinician-Administered PTSD Scale (CAPS; Blake 1990; Blake 1995), and the PTSD Symptom Scale - Interview (PSS-I; Foa 1993). Validated self-report measures include the PTSD Symptom Scale - Self-Report (PSS-SR; Foa 1993; Rothbaum 1990), Impact of Event Scale (IES; Horowitz 1979), Impact of Event Scale - Revised (IES-R; Weiss 1997), and PCL-5 (Bovin 2016), which is the self-reported PTSD Checklist for the Fifth Edition of the Diagnostic and Statistical Manual of Mental Disorders (DSM-5; APA 2013).

2. Treatment efficacy, depressive symptoms: response to treatment, determined by differences in scores for depressive symptoms, assessed by independent observer or self-report measures, including the Hospital Anxiety and Depression Scale 
(HADS; Zigmond 1983), Beck Depression Inventory (BDI; Beck 1961), Center for Epidemiologic Studies Depression Scale (CESD; Radloff 1977), Patient Health Questionnaire (PHQ; Spitzer 1999), and Hamilton Depression Rating Scale (HAM-D; Hamilton 1960).

3. Treatment acceptability: the number of participants who dropped out of the intervention (as distinct from attrition), including in studies of two intervention types and other assessments of acceptability (e.g. measures of patient/client satisfaction).

4. Adverse effects, such as counts of mortality, completed suicides, and attempted suicides, or worsening of symptoms (specifically, group differences on PTSD, depression, self-harm and suicidality - see below for tools), including those summarised in narrative form, or using a tool such as the Negative Effects Questionnaire (Rozental 2018). We will record whether or not studies made reference to this outcome.

\section{Secondary outcomes}

1. Anxiety symptoms, assessed with self-report scales such as the Beck Anxiety Inventory (BAl; Beck 1988), State-Trait Anxiety Inventory (STAI; Spielberger 1970), or Generalised Anxiety Disorder - Seven-item Scale (GAD-7; Kertz 2013; Spitzer 2006).

2. Dissociation symptoms, measured using instruments such as the Dissociative Experiences Scale (DES; Bernstein 1986), or the Dissociative Experiences Scale-II (DES-II; Bernstein 1986; Carlson 1993).

3. Global mental health functioning/distress, which is frequently measured by either the Global Severity Index (GSI), Positive Symptom Distress Index (PSDI) and Positive Symptom Total (PST) of the SCL-90-R (Derogatis 1983), or by the Behavior And Symptom Identification Scale (BASIS-32; Eisen 1999).

4. Feelings of guilt or self-blame (or both) experienced by survivors, measured by self-report tools such as the Trauma-Related Guilt Inventory (TRGI; Kubany 1996), Rape Attribution Questionnaire (RAQ; Frazier 2003), South African Stigma Scale (Singh 2011), Social Support Appraisal (SSA) scale (Vaux 1986), Rape Aftermath Symptom Test (RAST; Kilpatrick 1988), or Inventory of Interpersonal Problems (IPP; Horowitz 1988).

5. substance use, measured by a number of established scales, including the Michigan Alcoholism Screening Test (MAST; Selzer 1971), Drug Abuse Screening Test (DAST; Skinner 1982), Addiction Severity Index (ASI; McLellan 1980: McLellan 1992), Alcohol Use Inventory (AUI; Chang 2001), Drug Use Disorders Identification Test (DUDIT; Berman 2005), or the Alcohol Use Disorders Identification Test (AUDIT; Pradhan 2012).

6. Quality of life, which is commonly measured by self-report measures such as the WHO Quality of Life scale - Abbreviated Version (WHOQOL-BREF; Skevington 2004) and EuroQol-5 Dimensions (EQ-5D; Brooks 1996).

7. Self-harming or suicidality often measured by the Deliberate Self-Harm Inventory (DSHI; Gratz 2001), Self-Harm Behaviour Questionnaire (SHBQ; Guttierez 2001), or the Self-Injury Questionnaire (SIQ; Santa Mina 2006).

8. Sexual violence assessment, measured by instruments such as the Sexual Experiences Survey (SES; Koss 1987b) and the Abuse Assessment Screen (AAS) (Basile 2007; NSVRC 2011). These tools differ in terms of their method of delivery; their appropriateness for screening for females, males, or both; the setting in which screening is to occur; the total number of questions they con- tain; and the number of questions that are specific to sexual violence (Basile 2007; NSVRC 2011).

We will include all time points; however, the primary time point for treatment efficacy will be three months post-treatment. We will classify short-term time points as zero to six months, medium-term as six to 12 months, and long-term as 12 months or longer.

\section{Search methods for identification of studies}

\section{Electronic searches}

We will search the databases and trials registers listed below for published and unpublished studies. We will adapt the MEDLINE strategy in Appendix 2 for the other sources using appropriate indexing terms and syntax. We will not apply any limitations on publication date, place or language of any research; we will not exclude any potentially relevant studies and we will include research from different backgrounds and disciplines. The Information Specialist for Cochrane Developmental Psychosocial and Learning Problems will search all of the databases listed below, with the exception of the Common Mental Disorders Controlled Trials Register, which will be searched by the Information Specialist for Cochrane Common Mental Disorders.

1. Cochrane Central Register of Controlled Trials (CENTRAL; current issue) in the Cochrane Library, which includes the Developmental, Psychosocial and Learning Problems Specialised Register.

2. Cochrane Common Mental Disorders Controlled Trials Register (CCMDCTR; current to June 2016). See Appendix 3 for one of the core strategies (MEDLINE) used to populate CCMDCTR. Full details are available at $\mathrm{cmd}$.cochrane.org/specialised-register.

3. MEDLINE Ovid (1946 onwards).

4. MEDLINE In-Process \& Non-Indexed Citations Ovid (current issue).

5. MEDLINE Epub Ahead of Print Ovid (current issue).

6. Embase Ovid (1974 onwards).

7. CINAHL Plus EBSCOhost (Cumulative Index to Nursing and Allied Health Literature; 1937 onwards).

8. PsycINFO Ovid (1806 onwards).

9. ERIC EBSCOhost (Education Resources Information Center; 1966 onwards).

10.Social Policy and Practice Ovid (1890s onwards).

11.PTSDpubs Proquest (previously known as PILOTS; 1871 to present).

12.Cochrane Database of Systematic Reviews (current issue), a part of the Cochrane Library.

13. Web of Science Core Collection: Citation Indexes Clarivate Analytics (1970 onwards, Science Citation Index, Social Sciences Citation Index, Conference Proceedings Citation Index - Science and Conference Proceedings Citation Index - Social Science \& Humanities).

14.Epistemonikos (www.epistemonikos.org).

15.ClinicalTrials.gov (www.ClinicalTrials.gov).

16.WHO International Clinical Trials Registry Platform (ICTRP; apps.who.int/trialsearch).

17.Be Part of Research (replaced UK Clinical Trials Gateway; www.bepartofresearch.nihr.ac.uk/). 


\section{Searching other resources}

\section{Personal communication}

We will contact trialists and experts in the field regarding unpublished and ongoing research and to ask for further trial data. Those whom we will consider to be experts will be those who have conducted or authored studies and literature that are connected to the research (including Ronald Acierno, Ann Burgess, Enrique Echeburua, Edna Foa, Dean Kilpatrick, Patricia Resick, Heidi Resnick, and Barbara Rothbaum).

\section{Reference lists}

We will examine the reference lists of all included studies and relevant systematic reviews to identify additional studies from the electronic searches (for example, unpublished or in-press citations).

\section{Supplementary searches}

We will conduct a forward citation search of included studies using Web of Science.

\section{Data collection and analysis}

\section{Selection of studies}

Two review authors (NK and SB) will independently assess the titles and abstracts of all records identified through the searches against the inclusion criteria (Criteria for considering studies for this review), coding them as 'yes' (eligible), 'no' (not eligible) or maybe (potentially eligible or unclear). In the event of disagreements about inclusion, both review authors will assess and discuss the full article for relevance. If agreement cannot be reached by discussion, they will consult a third review author (LOD) as a mediator. Final decisions will be made by consensus.

We will retrieve full-text reports for selected abstracts and two pairs of review authors (NK and SB, LOD and LT) will independently assess each report against the inclusion criteria (Criteria for considering studies for this review). Studies will be identified for either inclusion or exclusion. We will contact study authors, as required, to decide whether the inclusion criteria have been met. We will record reasons for excluding ineligible studies. In the event of disagreements, we will consult a third review author (KB) as a mediator. Final decisions will be made by consensus.

We will identify and exclude duplicate records and will collate multiple reports that relate to the same study, so that each study, rather than each report, is the unit of interest in the review. We will record the selection process in sufficient detail to complete a four-phase (identification, screening, eligibility and included) PRISMA flow diagram for study collection (Moher 2009) and the 'Characteristics of excluded studies' tables.

\section{Data extraction and management}

We will use Covidence (Covidence 2018) as a platform to upload the included studies and extract data, and export data into Review Manager 5 (RevMan 5) (Review Manager 2014). We will generate a PRISMA diagram report. Review Manager will allow us to analyse the data and build the text, tables and figures for presenting the review.

We will pilot and refine the data collection form using the first five studies included in the review. Two pairs of review authors (NK paired with KB, LOD and GC) will independently extract data on key characteristics, methods and outcomes from each included study, and compare their results to identify differences. Where differences are identified, we will resolve them by consensus or by referral to another member of the review team (SB). When further clarification or missing data are needed from study authors, we will make all reasonable attempts to contact the study authors and obtain the relevant information.

Specifically, we will extract data on the following characteristics from each included study.

1. Methods: brief description of study design and randomisation method; dates or total duration of study; location of study.

2. Participants: baseline characteristics, including gender, age, ethnicity, sexual identity, markers of opportunity and deprivation; study setting; inclusion and exclusion criteria; number of eligible people recruited and assigned; number of dropouts; numbers analysed.

3. Interventions: number of intervention groups; type of psychosocial intervention; mode of delivery; frequency and duration of delivery; level of training of person delivering the intervention; relevant comparator intervention characteristics.

4. Outcomes: primary and secondary outcomes; outcome measures used; timing of outcome measurement.

5. Notes: funding for trial; notable conflicts of interest of trial authors.

One review author (NK) will transfer data into RevMan 5 (Review Manager 2014). Another review author (SB) will independently check the data extraction forms for accuracy and completeness.

\section{Assessment of risk of bias in included studies}

\section{Randomized parallel-group trials}

We will undertake our 'Risk of bias' assessment using Review Manager Web (RevMan Web) (Review Manager Web 2019) and according to Cochrane's revised 'Risk of bias' tool for randomised trials (RoB 2) (Sterne 2019). The study aims to assess the effect of assignment to intervention - the 'intention-to-treat' effect. We will assess the risk of bias for each result arising from studies that report our primary outcomes (i.e. treatment efficacy based on depression and PTSD). Depending on the availability of data for short-, medium- and longterm time points, we may apply RoB 2 to any result involving our primary outcome. Where there is indication of adverse effects in the form of worsening symptoms (self-harm and suicidality in addition to depression and PTSD), we will also apply RoB 2 to these results. Two pairs of review authors (NK and SB, LOD and GC) will independently undertake assessments. In the event of disagreements that cannot be resolved by discussion, we will consult another review author (GF or KB) as a mediator.

For a single trial result, we will respond to a series of 'signalling' questions covering five domains.

1. Risk of bias arising from the randomisation process.

2. Risk of bias due to deviations from the intended interventions (effect of assignment to intervention).

3. Risk of bias due to missing outcome data.

4. Risk of bias in measurement of the outcome.

5. Risk of bias in the selection of the reported result.. 
We will select one of the five response options to each question ('yes', 'probably yes', 'probably no', 'no' and 'no information'). We will use these responses to reach a judgement of low, high or some concerns. The final step will be to combine these responses for the five domains to reach an overall rating of low risk of bias, some or high risk of bias for the result. When considering treatment effects, we will take into account the risks of bias of the results contributing to that effect.

\section{Cluster-randomised parallel-group trials}

We will assess the risk of bias of cluster-randomised trials in line with Section 16.3.2 of the Cochrane Handbook for Systematic Reviews of Interventions (Higgins 2011a), assessing each study for risk of bias across the five domains listed below.

1. Bias arising from the randomisation process.

2. Bias due to deviation arising from intended interventions.

3. Bias due to missing outcome data.

4. Bias in the measurement of outcome.

5. Bias in the selection of the reported outcome.

We will also examine bias arising from identification or recruitment of individual participants within clusters.

\section{Randomised cross-over trials}

We do not expect to find cross-over trials; however, should we identify any, we will apply the same approach as recommended in Section 16.4.3 (Higgins 2011a), which essentially involves assessing the five domains above.

\section{Quasi-experimental}

In assessing the risk of bias in quasi-randomised studies, we will apply the same methods as those recommended for randomised trials, in line with Cochrane guidance (Higgins 2011b) and new guidance from Sterne and colleagues (Sterne 2019). Generally, we will judge such studies to be at high risk of bias arising from the randomisation process.

\section{Measures of treatment effect}

We will import the data for each study and outcome entered into Covidence (Covidence 2018) into RevMan 5 (Review Manager 2014), to perform meta-analyses and present results in graph form.

\section{Dichotomous data}

While the primary and secondary outcomes will usually be assessed with continuous measures, we expect that some investigators will present dichotomous data on these outcomes. We will require counts and percentages by trial arm for each study that reports dichotomous outcomes. Using the summary data, we will calculate the pooled risk ratio (RR) and $95 \%$ confidence intervals $(\mathrm{Cl})$ across the trials for each outcome. Where the data required to calculate the RR are neither available nor obtainable from the study authors, we will provide the findings as reported in the published paper.

\section{Continuous dato}

We will require means and standard deviations by trial arm for studies that report continuous outcomes. When studies have used the same continuous outcome measure, we will calculate mean differences (MD) with $95 \% \mathrm{Cl}$. When studies have used different outcome measures to assess the same construct, we will calculate standard- ised mean differences (SMD) and $95 \% \mathrm{Cl}$ as the measure of effect (Schünemann 2011). We expect that outcomes will have been measured with a range of tools (see Types of outcome measures) across studies, and that we will largely be calculating SMD. We will use Cohen's general rule of thumb to interpret effect sizes computed using the SMD, where 0.2 represents a small effect, 0.5 represents a medium effect, and 0.8 or larger represents a large effect (Cohen 1988). We will present conceptually distinct outcomes in separate forest plots. Where means and standard deviations are not available or obtainable from the study authors, we will provide the findings as reported in the published paper. We will use a narrative approach to describe continuous outcome data that do not have a normal distribution or are reported as medians and interquartile ranges, or both, given that meta-analysis assumes normality.

If it is necessary to combine dichotomous data and continuous data in a meta-analysis, we will need estimates of the standard error. Standard errors can be computed for all studies by entering the data into RevMan 5 as dichotomous and continuous outcome type data, as appropriate, and converting the $\mathrm{Cl}$ for the resulting log odds ratios and SMD into standard errors (Higgins 2011c). Once SMD (or log odds ratios) and their standard errors have been computed for all studies in the meta-analysis, we will combine them using the generic inverse-variance method in RevMan 5 (Review Manager 2014). Relating to outcomes where different scales can be used in reporting results, such as quality of life, we will use SMD to compile data.

\section{Unit of analysis issues}

\section{Cluster-randomised trials}

We do not anticipate any unit of analysis issues in this review. However, should we identify any cluster-randomised trials, we will adjust the standard errors or sample sizes using the method described in the Cochrane Handbook for Systematic Reviews of Interventions (Higgins 2017). The adjustment method requires the intra-class correlation coefficient (ICC). If the ICC is not available, we will use ICCS from analogous cluster-randomised trials. If analogous studies are not available, we will use a series of plausible values in a sensitivity analysis (see Sensitivity analysis).

\section{Studies with multiple treatment groups}

If studies compare multiple eligible experimental interventions with a single control group, we will split the control group to enable pairwise comparisons. If studies use multiple control groups, we will combine the control groups to compare them to the experimental intervention group.

\section{Dealing with missing data}

Where data are missing, we will follow the recommendations outlined in the Cochrane Handbook for Systematic Reviews of Interventions (Higgins 2011a). We will classify data as either 'missing at random' or 'not missing at random'. Where we consider data to be missing at random, we will analyse the available data. For data that we consider not missing at random, we will make every effort to contact study authors to gather the missing information. We will ask questions in an open-ended manner to prevent the skewing of responses (Higgins 2011a). We will document all correspondence with study authors. It will not be possible to use analytical methods to handle missing data as we will only collect summary data from the studies; we will not source individual level data from the 
study authors (Egger 2001). We will highlight any suppositions that we make during our analysis when data are unavailable. We will estimate the log rank statistics where these are not published in an article, and we will use previously reported methods, where applicable (Parmar 1998; Tierney 2007). We will address the potential impact of missing outcome data in the 'Risk of bias' assessment. If appropriate, we will perform a sensitivity analysis to assess the impact of the missing information on our results (see Sensitivity analysis), using the methods described in theCochrane Handbook for Systematic Reviews of Interventions (Higgins 2011a): section 16.2.2 for dichotomous outcomes and section 16.2.3 for continuous outcomes.

\section{Assessment of heterogeneity}

Variability in the participants, interventions and outcomes studied may be described as clinical heterogeneity; variability in study design and risk of bias may be described as methodological heterogeneity. Variability in the intervention effects being evaluated in the different studies is known as statistical heterogeneity. It is a consequence of clinical or methodological heterogeneity, or both, among the studies and manifests in the observed intervention effects being more different from each other than one would expect due to random error (chance) alone.

We will identify sources of clinical heterogeneity by constructing tables to summarise studies in terms of participants, setting, type of intervention, intervention delivery (e.g. group or individual, number of sessions) and outcomes examined. Where studies are similar, we will conduct further analyses, initially by reviewing the consistency of the results across studies using graphical representations (Egger 1997). To initially identify the heterogeneity/inconsistency of the whole network, we will use the Q statistic, separating the studies based on whether they share the same design or not. We will assess statistical heterogeneity with the $\mathrm{Chi}^{2}$ test, which will provide us with evidence of variation in effects, disregarding the effect of chance. The $\mathrm{Chi}^{2}$ test is ineffective for analysing heterogeneity in studies with only a small number of participants or trials, so we will set our P value at 0.10 (Deeks 2017), and assess heterogeneity using the $\mathrm{I}^{2}$ statistic, which will find the percentage of variability due to heterogeneity outside of the effect of chance (Higgins 2003).

We assume that some statistical heterogeneity is inevitable, and hence, will evaluate heterogeneity using the $1^{2}$ statistic (Deeks 2017). We will interpret the observed value of $\mathrm{I}^{2}$ using the guide given in Section 9.5.2 of the Cochrane Handbook for Systematic Reviews of Interventions (Deeks 2017), where 0\% to $40 \%$ might not be important, $30 \%$ to $60 \%$ may represent moderate heterogeneity, $50 \%$ to $90 \%$ may represent substantial heterogeneity, and $75 \%$ to $100 \%$ shows considerable heterogeneity. We will take into consideration the size and direction of effects and the strength of evidence for heterogeneity using the $\mathrm{Chi}^{2}$ test and the $95 \% \mathrm{Cl}$ for $\mathrm{I}^{2}$.

Where there is evidence for statistical heterogeneity, we will use the strategies outlined in Section 9.5.3 of the Cochrane Handbook for Systematic Reviews of Interventions (Deeks 2017), to identify potential sources of heterogeneity among the results of the studies. In particular, we will explore differences in the characteristics of the studies or other factors as possible explanations for heterogeneity in the results. We will summarise any differences identified in the narrative summary. The significance of the $1^{2}$ statistics observed will rely upon the effects of treatment and the quality of evidence suggesting heterogeneity.
We will use RevMan 5 (Review Manager 2014) to produce forest plots and calculate tau ${ }^{2}$, the between-trial variance in a random-effects meta-analysis (Deeks 2017; Review Manager 2014). To understand the intervention effects, we will use tau ${ }^{2}$ to identify a range for the primary outcome. We will use the Cochrane Handbook for Systematic Reviews of Interventions as a guideline throughout this process (Deeks 2017).

\section{Assessment of reporting biases}

We will attempt to locate the protocols or study records (or both) in trial registries of the RCTs included in the review. Where the protocol is available, we will compare its outcomes against the published report; and where the protocol cannot be found, we will compare the outcomes included in the methods section of the trial report to the reported results. We will identify outcome reporting bias where outcomes are included in the methods but not reported (Pocock 1987; Tannock 1996).

If there are 10 or more studies, we will construct funnel plots to investigate associations between effect size and study precision (which is closely related to sample size) (Egger 1997). We will also apply Egger's regression asymmetry test to funnel plots to test for funnel plot asymmetry (Egger 1997). Such an association could be due to publication or related biases, or due to systematic differences between small and large studies. If we identify an association, we will examine the clinical diversity of the studies as a possible explanation. If appropriate, we will also conduct a sensitivity analysis to determine whether assumptions about the effect of the bias impact the estimated treatment effect and the conclusions of the review (see Sensitivity analysis).

\section{Data synthesis}

We will perform a meta-analysis if there are sufficient data and it is meaningful to pool the data across studies; for instance, the treatments, participants and the underlying clinical measures are similar enough for pooling to make sense. Our decision to perform a meta-analysis will be determined by the comparability of populations, denominators and interventions (clinical heterogeneity); the comparability of the duration of follow-up (methodological heterogeneity); and the comparability of outcomes. We will use a random-effects model to analyse the data across the studies. The Mantel-Haenszel method, a default program in RevMan 5 (Review Manager 2014), can take account of few events or small study sizes, and can be used with random-effects models.

We will stratify results for the main comparison (psychosocial interventions versus inactive controls, such as usual care, no treatment, delayed provision of psychological interventions (or waiting-list conditions) or pharmacological treatment only, and minimal interventions such as information provision) by type of therapy (categories 1 to 7 listed under Types of interventions), where there are sufficient numbers of studies of the same intervention type, comparison arm and reporting the same outcome. For other comparisons, comparing two experimental interventions (i.e. an intervention from one category against an intervention from another category), we will again require two or more studies comparing similar experimental interventions using similar outcomes.

If it is inappropriate to combine the data in a meta-analysis (on account of insufficient studies or data), we will report the effect sizes with $95 \% \mathrm{Cl}$ or standard errors of individual studies, and provide 
a narrative, rather than quantitative, summary of the findings that addresses the following aspects.

1. What is the direction of effect?

2. What is the size of effect?

3. Is the effect consistent across studies?

\section{Subgroup analysis and investigation of heterogeneity}

We are keen to investigate intervention effects according to subsets of participants and for subsets of studies, and we will perform the subgroup analyses set out below. However, we are aware that subgroup analyses of subsets of participants are challenged because sufficient details to extract data about separate participant types are seldom published in reports.

1. Category of intervention (e.g. CBT, behavioural therapies, headto-head comparisons)

2. Participant characteristics (e.g. gender, ethnicity, time to treatment, symptom load, and types of trauma exposure)

3. Intensity of intervention (e.g. up to four sessions, five or more sessions)

4. Mode of intervention delivery (e.g. individual versus group)

5. Setting of recruitment or intervention delivery (healthcare, community, police-led, charity-led)

Participant characteristics have been identified as integral to subgroup analyses, as there may be differences in the efficacy of treatments for different groups of individuals. Recruitment setting has also been identified as important, as there may be differences between survivors recruited via healthcare as opposed to police-led and criminal justice or charity environments. Intensity of interventions are also of interest; we might, for example, compare outcomes from intense psychological therapies versus interventions oriented towards provision of psychosocial support. Finally, it will be important to stratify analyses by type of intervention given their distinct mechanisms and theoretical underpinnings. The characteristics related to participants, settings and interventions will have important practical implications for our review findings and recommendations.

We will use a simple approach, described in Chapter 9.6.3 of the Cochrane Handbook for Systematic Reviews of Interventions (Deeks 2017), to investigate whether there is a difference in the intervention effect between the subgroups. As described in Chapter 9.6.4 of the Cochrane Handbook for Systematic Reviews of Interventions (Deeks 2017), meta-regression is an extension to subgroup analyses that allows the effect of continuous as well as categorical characteristics to be investigated, and, in principle, allows the effects of multiple factors to be investigated simultaneously. Generally, meta-regression should not be considered when there are fewer than 10 studies in a meta-analysis. If there are more than 10 studies available, we will use meta-regression techniques recommended for STATA (Harbord 2004; Stata 2017).

If we identify a considerable degree of heterogeneity (75\% to $100 \%)$, we will first check the data for errors. If the data are correct, we will conduct a sensitivity analysis by excluding certain studies from the existing meta-analysis, to assess the influence of the studies on the degree of heterogeneity (see Sensitivity analysis).

\section{Sensitivity analysis}

We will base our primary analyses on available data from all included studies relevant to the comparison of interest. However, in order to examine any effects of methodological decisions on the overall outcome, we will perform sensitivity analyses provided there are sufficient numbers of studies. These sensitivity analyses may include the following.

1. Re-analysis of the data excluding studies with results at high risk of bias.

2. Re-analysis of the data excluding studies with missing outcome data.

3. Re-analysis of the data excluding other identified studies of low methodological quality.

Additional sensitivity analyses may be required if particular issues related to the studies under review arise.

\section{'Summary of findings' table}

We will create our 'Summary of findings' table(s) using GRADEpro GDT (GRADEpro GDT 2015) and following standard methods described in the Cochrane Handbook for Systematic Reviews of Interventions (Schünemann 2017). The table(s) will provide key information concerning the quality of evidence, the magnitude of effect of the interventions examined, and the sum of available data on primary outcomes. The table(s) will include details relating to the participants, interventions, comparisons, outcomes (PICO), settings, length of the follow-up, and outcome measurement.

The key comparison for the 'Summary of findings' table(s) will be impact of psychosocial interventions versus inactive controls on treatment efficacy. For each outcome, we will present standardised effect size estimates and $95 \% \mathrm{Cl}$. The primary outcomes for the review are: treatment efficacy measured by group differences on PTSD symptoms and on depressive symptoms. The table will present treatment acceptability based on worsening of the primary treatment outcomes and self-harm and suicidality, and intervention dropout rates. It will also present adverse outcomes.

It is recognised that the main comparison combines all intervention types in one and that it may be more useful to stakeholders to understand effects by type. Thus, depending on availability of data, we will stratify results using primary outcomes for CBT versus inactive controls and behavioural therapies versus inactive controls, presenting these in additional tables.

We will assess the certainty of the evidence using the GRADE approach and will include the results of this assessment in the 'Summary of findings' table(s). The level of certainty will be defined by five factors: risk of bias; indirectness of factors (such as evidence, population, control, intervention and outcomes); inconsistency of results; imprecision of results (and large $\mathrm{Cl}$ ); and a high likelihood of publication bias. We will downgrade all evidence by one level for a single factor up to a maximum of three levels for all factors. The final grade will be determined by how likely the effect can be predicted. We will assess the certainty of the evidence on a four-point scale, ranging from high (the real effect is close to what will be predicted) to very low (what actually happens is significantly varied from the predicted effect) (Schünemann 2017).

We will create the 'Summary of findings' table(s) after we have entered the data into RevMan 5 (Review Manager 2014), written up our 
results and conducted the risk of bias assessment, but before writing our abstract, discussion and conclusions, to allow the opportunity to consider the impact of risk of bias in the studies contributing to each outcome on the mean treatment effect and our confidence in these findings.

\section{ACKNOWLEDGEMENTS}

This Cochrane Review is part of a larger study about healthcare for sexual violence: Multidisciplinary Evaluation of Sexual Assault Referral Centres for better Health (MESARCH). The research is funded by an institutional (Coventry University) research grant (project number 16/117/04) from the National Institute for Health Research (NIHR) Health Service and Delivery Research Programme.

We would like to thank members of the MESARCH Study Steering Committee, in particular Gillian Finch of our Lived Experiences Group, for input into the development of this protocol.
We would also like to thank members of the Cochrane Developmental, Psychosocial and Learning Problems review group for their guidance in developing this protocol and the following reviewers for their contributions in refining it: Dee Shneiderman; Lisa Fedina PhD, University of Michigan, School of Social Work; Anao Zhang, University of Michigan School of Social Work; Dr Ben Carter, Senior Lecturer in Biostatistics and KCTU Mental Health Statistics Group Lead, King's College London; and Lindsay DG Thomson, Professor of Forensic Psychiatry, University of Edinburgh, Medical Director, The State Hospitals Board for Scotland, and Director of Forensic Network and School of Forensic Mental Health.

Finally, we would like to thank the Cochrane Editorial and Methods Department for the opportunity to participate in a pilot of the new risk of bias tool (RoB 2), and their support in preparing this Protocol.

Disclaimer: The views expressed herein are those of the review authors and not necessarily those of the NHS, the NIHR or the Department of Health and Social Care. 


\section{R E F E R E N C E S}

\section{Additional references}

\section{APA 2013}

American Psychiatric Association. Diagnostic and Statistical Manual of Mental Disorders (DSM-5). 5th Edition. Arlington (VA): American Psychiatric Publishing, 2013.

\section{Arroyo 2017}

Arroyo K, Lundahl B, Butters R, Vanderloo M, Wood DS. Shortterm interventions for survivors of intimate partner violence: a systematic review and meta-analysis. Trauma, Violence \& Abuse 2017;18(2):155-71. [DOI: 10.1177/1524838015602736; PUBMED: 26335794]

\section{Basile 2007}

Basile KC, Hertz MF, Back SE. Intimate Partner Violence and Sexual Violence Victimization Assessment Instruments for Use in Healthcare Settings. 1. Atlanta (GA): Centers for Disease Control and Prevention, National Center for Injury Prevention and Control, 2007.

\section{Beck 1961}

Beck AT, Ward CH, Mendelson M, Mock J, Erbaugh J. An inventory for measuring depression. Archives of General Psychiatry 1961;4(6):561-71. [DOI: 10.1001/ archpsyc.1961.01710120031004; PUBMED: 13688369]

\section{Beck 1985}

Beck AT, Emery G, Greenberg RL. Anxiety Disorders and Phobias: A Cognitive Perspective. New York (NY): Basic Books, 1985.

\section{Beck 1988}

Beck AT, Steer RA, Garbin MG. Psychometric properties of the Beck Depression Inventory: twenty-five years of evaluation. Clinical Psychology Review 1988;8(1):77-100. [DOI: 10.1016/0272-7358(88)90050-5]

\section{Berg 2002}

Berg SH. The PTSD diagnosis: is it good for women?. Affilia 2002;17(1):55-68. [DOI: 10.1177/0886109902017001004]

\section{Berman 2005}

Berman AH, Bergman H, Palmstierna T, Schlyter F. Evaluation of the Drug Use Disorders Identification Test (DUDIT) in criminal justice and detoxification settings and in a Swedish population sample. European Addiction Research 2005;11(1):22-31. [DOI: 10.1159/000081413; PUBMED: 15608468]

\section{Bernstein 1986}

Bernstein EM, Putnam FW. Development, reliability, and validity of a dissociation scale. Journal of Nervous and Mental Disease 1986;174(12):727-35. [PUBMED: 3783140]

\section{Bisson 2013}

Bisson JI, Roberts NP, Andrew M, Cooper R, Lewis C. Psychological therapies for chronic post-traumatic stress disorder (PTSD) in adults. Cochrane Database of Systematic Reviews 2013, Issue 12. [DOI: 10.1002/14651858.CD003388.pub4; PUBMED: 24338345]

\section{Blake 1990}

Blake DD, Weathers FW, Nagy LM, Kaloupek DG, Klauminzer G, Charney DS, et al. A clinician rating scale for assessing current and lifetime PTSD: the CAPS-1. Behavior Therapist 1990;13:1878.

\section{Blake 1995}

Blake DD, Weathers FW, Nagy LM, Kaloupek DG, Gusman FD, Charney DS, et al. The development of a clinician-administered PTSD scale. Journal of Traumatic Stress 1995;8(1):75-90.

\section{BluePages 2012}

BluePages. Treatment for depression. bluepages.anu.edu.au/ treatments/ what ${ }^{\prime}$ works/psychological ${ }^{~}$ treatments/ (accessed 20 August 2012).

\section{Boudewyns 1996a}

Boudewyns PA. Post-traumatic stress disorder: conceptualization and treatment. In: Hersen M, Eisler RM, Miller PM editor(s). Progress in Behavior Modification. Vol. 30, Pacific Grove (CA): Brooks/Cole Publishing Co, 1996:165-89.

\section{Boudewyns 1996b}

Boudewyns PA, Hyer LA. Eye movement desensitization and reprocessing (EMDR) as treatment for posttraumatic stress disorder (PTSD). Clinical Psychology \& Psychotherapy 1996;3(3):185-95. [DOI: 10.1002/ (SICI)1099-0879(199609)3:3<185::AID-CPP101>3.0.CO;2-0]

\section{Bovin 2016}

Bovin MJ, Marx BP, Weathers FW, Gallagher MW, Rodriguez P, Schnurr PP, et al. Psychometric properties of the PTSD Checklist for Diagnostic and Statistical Manual of Mental Disorders Fifth Edition (PCL-5) in veterans. Psychological Assessment 2016;28(11):1379-91. [DOI: 10.1037/pas0000254; PUBMED: 26653052 ]

\section{Brennan 2012}

Brennan J, Kuhns LM, Johnson AK, Belzer M, Wilson EC, Garofalo R. Syndemic theory and HIV-related risk among young transgender women: the role of multiple, co-occurring health problems and social marginalization. American Journal of Public Health 2012;102(9):1751-7.

\section{Brooks 1996}

Brooks R. EuroQol: the current state of play. Health Policy 1996;37(1):53-72. [PUBMED: 10158943]

\section{Brotto 2012}

Brotto LA, Seal BN, Rellini A. Pilot study of a brief cognitive behavioral versus mindfulness-based intervention for women with sexual distress and a history of childhood sexual abuse. Journal of Sex \& Marital Therapy 2012;38(1):1-27. [DOI: 10.1080/0092623X.2011.569636; PUBMED: 22268979]

\section{Buchwald 1993}

Buchwald E, Fletcher P, Roth M, editor(s). Transforming a Rape Culture. Minneapolis (MN): Milkweed Editions, 1993. 


\section{Burgess 1974}

Burgess AW, Holmstrom LL. Rape trauma syndrome. American Journal of Psychiatry 1974;131(9):981-6. [DOI: 10.1176/ ajp.131.9.981]

\section{Burt 1998}

Burt MR. Rape myths. In: Odem ME, Clay-Warner J editor(s). Confronting Rape and Sexual Assault. Lanham (MD): Rowman \& Littlefield, 1998:129-44.

\section{Butler 2006}

Butler AC, Chapman JE, Forman EM, Beck AT. The empirical status of cognitive-behavioral therapy: a review of metaanalyses. Clinical Psychology Review 2006;26(1):17-31. [DOI: 10.1016/j.cpr.2005.07.003; PUBMED: 16199119]

\section{Campbell 1998}

Campbell R. The community response to rape: victims' experiences with the legal, medical, and mental health systems. American Journal of Community Psychology 1998;26(3):355-79. [PUBMED: 9726113]

\section{Campbell 1999}

Campbell JC, Soeken KL. Forced sex and intimate partner violence: effects on women's risk and women's health. Violence Against Women 1999;5(9):1017-35. [DOI: $10.1177 / 1077801299005009003]$

\section{Campbell 2001}

Campbell R, Martin PY. Services for sexual assault survivors: the role of rape crisis centers. In: Renzetti C, Edleson JL, Kennedy Bergen R editor(s). Sourcebook on Violence Against Women. Thousand Oaks (CA): Sage Publications, Inc., 2001:227-41.

\section{Campbell 2002}

Campbell JC. Health consequences of intimate partner violence. Lancet 2002;359(9314):1331-6. [DOI: 10.1016/ S0140-6736(02)08336-8; PUBMED: 11965295]

\section{Campbell 2004}

Campbell R, Sefl T, Ahrens CE. The impact of rape on women's sexual health risk behaviours. Health Psychology 2004;23(1):67-74. [DOI: 10.1037/0278-6133.23.1.67; PUBMED: 14756605]

\section{Campbell 2009}

Campbell R, Dworkin E, Cabral G. An ecological model of the impact of sexual assault on women's mental health. Trauma, Violence \& Abuse 2009;10(3):225-46. [DOI: 10.1177/1524838009334456; PUBMED: 19433406]

\section{Carlson 1993}

Carlson EB, Putnam FW. An update on the Dissociative Experiences Scale. Dissociation: Progress in the Dissociative Disorders 1993;6(1):16-27.

\section{CDC 2004}

Centers for Disease Control and Prevention (CDC). Sexual Violence Prevention: Beginning the Dialogue. Altanta (GA): Centers for Disease Control and Prevention, 2004.

\section{Chang 2001}

Chang I, Lapham SC, Wanberg KW. Alcohol Use Inventory: screening and assessment of first-time driving-while-impaired offenders. I. Reliability and profiles. Alcohol and Alcoholism 2001;36(2):112-21. [PUBMED: 11259207]

\section{Cohen 1988}

Cohen J. Statistical Power Analysis for the Behavioral Sciences. New York (NY): Routledge, 1988.

\section{Cohen 2005}

Cohen JA, Mannarino AP, Knudsen K. Treating sexually abused children: 1 year follow-up of a randomised controlled trial. Child Abuse \& Neglect 2005;29(2):135-45. [DOI: 10.1016/ j.chiabu.2004.12.005; PUBMED: 15734179]

\section{Covidence 2018 [Computer program]}

Veritas Health Innovation. Covidence. Version accessed 5 December 2018. Melbourne, Australia: Veritas Health Innovation, 2018.

\section{Coxell 2010}

Coxell AW, King MB. Male victims of rape and sexual abuse. Sexual and Relationship Therapy 2010;25(4):380-91. [DOI: 10.1080/14681994.2010.518725]

\section{CPS 2002}

Crown Prosecution Service, Department of Health, Home Office. Provision of Therapy for Vulnerable or Intimidated Witnesses Prior to a Criminal Trial: Practice Guidance. London (UK): Home Office Communications Directorate, 2002.

\section{Cryer 1980}

Cryer L, Beutler L. Group therapy: an alternative treatment approach for rape victims. Journal of Sex \& Marital Therapy 1980;6(1):40-6. [DOI: 10.1080/00926238008404244; PUBMED: 7381947]

\section{Deblinger 2001}

Deblinger E, Stauffer LB, Steer RA. Comparative efficacies of supportive and cognitive behavioral group therapies for young children who have been sexually abused and their nonoffending mothers. Child Maltreatment 2001;6(4):332-43. [DOI: 10.1177/1077559501006004006; PUBMED: 11675816]

\section{Deeks 2017}

Deeks JJ, Higgins JPT, Altman DG, editor(s), Cochrane Statistical Methods Group. Chapter 9: Analysing data and undertaking meta-analyses. In: Higgins JPT, Churchill R, Chandler J, Cumpston MS, editor(s). Cochrane Handbook for Systematic Reviews of Interventions version 5.2.0 (updated June 2017). The Cochrane Collaboration, 2017. Available from training.cochrane.org/handbook.

\section{Derogatis 1983}

Derogatis RL. SCL-90-R. Administration, Scoring \& Procedures Manual-II for the R(evised) Version and Other Instruments of the Psychopathology Rating Scales Series. 2nd Edition. Towson (MD): Clinc Psychometric Research, 1983. 


\section{Dobson 2009}

Dobson KS. Handbook of Cognitive-Behavioral Therapies. 3rd Edition. New York (NY): Guilford Press, 2009.

\section{Dognin 2017}

Dognin J, Sedlander E, Jay M, Ades V. Group education sessions for women veterans who experienced sexual violence: qualitative findings. Families, Systems \& Health 2017;35(3):360-72. [DOI: 10.1037/fsh0000262; PUBMED: 28414479]

\section{Earles 2015}

Earles JL, Vernon LL, Yetz JP. Equine-assisted therapy for anxiety and posttraumatic stress symptoms. Journal of Traumatic Stress 2015;28(2):149-52. [DOI: 10.1002/jts.21990; PUBMED: 25782709]

\section{Egger 1997}

Egger M, Davey Smith G, Schneider M, Minder C. Bias in meta-analysis detected by a simple, graphical test. $B M J$ 1997;315(7109):629-34. [DOI: 10.1136/bmj.315.7109.629; PMC2127453; PUBMED: 9310563 ]

\section{Egger 2001}

Egger M, Davey Smith G, Altman EG. Systematic Reviews in Health care: Meta-analysis in Context. 2nd Edition. London (UK): BMJ Publishing Group, 2001.

\section{Eisen 1999}

Eisen SV, Culhane MA. Behavior And Symptom Identification Scale (BASIS-32). In: Maruish ME editor(s). The Use of Psychological Testing for Treatment Planning and Outcomes Assessment. Vol. 1: General Considerations, Mahwah (NJ): Lawrence Erlbaum Associates, 1999:759-90.

\section{Foa 1986}

Foa EB, Kozak MJ. Emotional processing of fear: exposure to corrective information. Psychological Bulletin 1986;99(1):20-35. [PUBMED: 2871574]

\section{Foa 1991}

Foa EB, Rothbaum BO, Riggs DS, Murdock TB. Treatment of posttraumatic stress disorder in rape victims: a comparison between cognitive behavioral procedures and counselling. Journal of Consulting and Clinical Psychology 1991;59(5):715-23. [PUBMED: 1955605]

\section{Foa 1993}

Foa EB, Riggs DS, Dancu CV, Rothbaum BO. Reliability and validity of a brief instrument for assessing post-traumatic stress disorder. Journal of Traumatic Stress 1993;6(4):459-73. [DOI: 10.1002/jts.2490060405]

\section{Foa 1994}

Foa EB, Riggs DS. Posttraumatic stress disorder and rape. In: Pynoos RS editor(s). Posttraumatic Stress Disorder: A Clinical Review. Lutherville (MD): Sidran Press, 1994:133-58.

\section{Frazier 2003}

Frazier PA. Perceived control and distress following sexual assault: a longitudinal test of a new model. Journal of
Personality and Social Psychology 2003;84(6):1257-69.

[PUBMED: 12793588]

\section{Freeman 2005}

Freeman A, Felgoise SH, Nezu AM, Nezu CM, Reinecke MA, editor(s). Encyclopedia of Cognitive Behavior Therapy. New York (NY): Springer, 2005.

\section{Gilfus 1999}

Gilfus ME. The price of the ticket: a survivor-centered appraisal of trauma theory. Violence Against Women 1999;5(11):1238-57.

\section{Gillies 2016}

Gillies D, Maiocchi L, Bhandari AP, Taylor F, Gray C, O'Brien L. Psychological therapies for children and adolescents exposed to trauma. Cochrane Database of Systematic Reviews 2016, Issue 10. [DOI: 10.1002/14651858.CD012371; PUBMED: 27726123 ]

\section{Goodman 1993}

Goodman LA, Koss MP, Russo NF. Violence against women: physical and mental health effects. Part I: research findings. Applied and Preventative Psychology 1993;2(2):79-89. [DOI: 10.1016/S0962-1849(05)80114-3]

\section{GRADEpro GDT 2015 [Computer program]}

McMaster University (developed by Evidence Prime). GRADEpro GDT. Version accessed 5 December 2018. Hamilton (ON): McMaster University (developed by Evidence Prime), 2015.

\section{Gratz 2001}

Gratz KL. Measurement of deliberate self-harm: preliminary data on the Deliberate Self-Harm Inventory. Journal of Psychopathology and Behavioral Assessment 2001;23(4):253-63. [DOI: 10.1023/A:1012779403943]

\section{Guina 2016}

Guina J, Nahhas RW, Kawalec K, Farnsworth S. Are gender differences in DSM-5 PTSD symptomatology explained by sexual trauma?. Journal of Interpersonal Violence 2016 Nov 1 [Epub ahead of print]. [DOI: 10.1177/0886260516677290; PUBMED: 27827321]

\section{Guttierez 2001}

Guttierez PM, Osman A, Barrios FX, Kopper BA. Development and initial validation of the Self-Harm Behavior Questionnaire. Journal of Personality Assessment 2001;77(3):475-90.

\section{Hamilton 1960}

Hamilton M. A rating scale for depression. Journal of Neurology, Neurosurgery and Psychiatry 1960;23(1):56-62. [DOI: 10.1136/ jnnp.23.1.56; PMC495331; PUBMED: 14399272 ]

\section{Harbord 2004 [Computer program]}

Harbord R, Higgins J. METAREG: Stata module to perform metaanalysis regression. Version (revised 05 Jan 2009). Boston College, Department of Economics (MA): Statistical Software Components S446201, 2004.

\section{Harte 2013}

Harte CB, Hamilton LD, Meston CM. Predictors of attrition from an expressive writing intervention for sexual abuse 
survivors. Journal of Child Sexual Abuse 2013;22(7):842-57. [DOI: 10.1080/10538712.2013.830670; PUBMED: 24125085]

\section{Harvey 1996}

Harvey MR. An ecological view of psychological trauma and trauma recovery. Journal of Traumatic Stress 1996;9(1):3-23. [PUBMED: 8750448]

\section{Hayes 2006}

Hayes SC, Luoma JB, Bond FW, Masuda A, Lillis J. Acceptance and commitment therapy: model, processes and outcomes. Behaviour Research and Therapy 2006;44(1):1-25. [DOI: 10.1016/ j.brat.2005.06.006; PUBMED: 16300724]

\section{Herman 1992}

Herman JL. Complex PTSD: a syndrome in survivors of prolonged and repeated trauma. Journal of Traumatic Stress 1992;5(3):377-91. [DOI: 10.1002/jts.2490050305]

\section{Hetrick 2010}

Hetrick SE, Purcell R, Garner B, Parslow R. Combined pharmacotherapy and psychological therapies for post traumatic stress disorder (PTSD). Cochrane Database of Systematic Reviews 2010, Issue 7. [DOI: 10.1002/14651858.CD007316.pub2; PUBMED: 20614457]

\section{Higgins 2011a}

Higgins JPT, Deeks JJ, Altman DG, editor(s), Cochrane Statistical Methods Group. Chapter 16: Special topics in statistics. In: Higgins JP, Green S, editor(s). Cochrane Handbook for Systematic Reviews of Interventions Version 5.1.0 (updated March 2011). The Cochrane Collaboration, 2011. Available from handbook.cochrane.org.

\section{Higgins 2011b}

Reeves BC, Deeks JJ, Higgins JPT, Wells GA, Cochrane Non-Randomised Studies Methods Group. Chapter 13: Including non-randomized studies. In: Higgins JP, Green S, editor(s). Cochrane Handbook for Systematic Reviews of Interventions Version 5.1.0 (updated March 2011). The Cochrane Collaboration, 2011.. Available from handbook.cochrane.org.

\section{Higgins 2011c}

Higgins JPT, Deeks JJ, editor(s). Chapter 7: Selecting studies and collecting data. In: Higgins JP, Green S, editor(s). Cochrane Handbook for Systematic Reviews of Interventions Version 5.1.0 (updated March 2011). The Cochrane Collaboration, 2011. Available from handbook.cochrane.org.

\section{Higgins 2017}

Higgins JPT, Altman DG, Sterne JAC, editor(s). Chapter 8: Assessing risk of bias in included studies. In: Higgins JPT, Churchill R, Chandler J, Cumpston MS, editor(s). Cochrane Handbook for Systematic Reviews of Interventions Version 5.2.0 (updated June 2017). The Cochrane Collaboration, 2017. Available from training.cochrane.org/handbook.

\section{Home Office 2005}

Home Office. The economic and social costs of crime against individuals and households 2003/04: Home Office online report 30/05. webarchive.nationalarchives.gov.uk/20110218140138/ http://rds.homeoffice.gov.uk/rds/pdfs05/rdsolr3005.pdf (accessed 3 January 2019).

\section{Home Office 2017}

Home Office. The role of the independent sexual violence adviser: essential elements. bit.ly/2En4iOv (accessed 3 January 2019).

\section{Horowitz 1979}

Horowitz M, Wilner N, Alvarez W. Impact of Event Scale: a measure of subjective stress. Psychosomatic Medicine 1979;41(3):209-18. [PUBMED: 472086]

\section{Horowitz 1988}

Horowitz LM, Rosenberg SE, Baer BA, Ureño G, Villaseñor VS. Inventory of Interpersonal Problems: psychometric properties and clinical applications. Journal of Consulting and Clinical Psychology 1988;56(6):885-92. [PUBMED: 3204198]

\section{IOM 2015}

Institute of Medicine. Psychosocial Interventions for Mental and Substance Use Disorders: A Framework for Establishing Evidence-Based Standards. Washington (DC): The National Academies Press, 2015.

\section{Jewkes 2002}

Jewkes R, Sen P, Garcia-Moreno C. Sexual violence. In: Krug EG, Dahlberg LL, Mercy JA, Zwi AB, Lozano R editor(s). World Report on Violence and Health. Geneva $(\mathrm{CH})$ : World Health Organization, 2002:147-81.

\section{Jewkes 2011}

Jewkes R, Sikweyiya Y, Morrell R, Dunkle K. Gender inequitable masculinity and sexual entitlement in rape perpetration South Africa: findings of a cross-sectional study. PLOS One 2011;6(12):e29590. [DOI: 10.1371/journal.pone.0029590; PMC3247272; PUBMED: 22216324]

\section{Kelly 1966}

Kelly JG. Ecological constraints on mental health services. American Psychologist 1996;21(6):535-9. [DOI: 10.1037/ h0023598]

\section{Kelly 1968}

Kelly JG. Towards an ecological conception of preventive interventions. Research Contributions from Psychology to Community Mental Health. Carter JW Jr. New York (NY): Behavioral Publications, 1968:75-99. [ERIC: ED022220; files.eric.ed.gov/fulltext/ED022220.pdf]

\section{Kelly 1971}

Kelly JG. Qualities for the community psychologist. American Psychologist 1971;26(10):897-903. [DOI: 10.1037/h0032231]

\section{Kertz 2013}

Kertz S, Bigda-Peyton J, Bjorgvinsson T. Validity of the Generalized Anxiety Disorder-7 Scale in an acute psychiatric sample. Clinical Psychology \& Psychotherapy 2013;20(5):456-64. [DOI: 10.1002/cpp.1802; PUBMED: 22593009] 


\section{Kilpatrick 1983}

Kilpatrick DG, Veronen LJ. Treatment for rape-related problems: crisis intervention is not enough. In: Cohen LH, Claiborn WL, Spector CA editor(s). Crisis Intervention. New York (NY): Human Sciences Press, 1983:165-85.

\section{Kilpatrick 1988}

Kilpatrick DG. Rape Aftermath Symptom Test. In: Hersen M, Bellack AS editor(s). Dictionary of Behavioral Assessment Techniques. Oxford (UK): Pergamon Press, 1988:366-7.

\section{Koss 1987 a}

Koss MP, Harvey MR. The Rape Victim: Clinical and Community Approaches to Treatment. Lexington (MA): Stephen Greene Press, 1987.

\section{Koss 1987b}

Koss MP, Gidycz CA, Wisniewski N. The scope of rape: incidence and prevalence of sexual aggression and victimization in a national sample of higher education students. Journal of Consulting and Clinical Psychology 1987;55(2):162-70. [PUBMED: 3494755]

\section{Koss 1991}

Koss MP, Harvey MR. The Rape Victim: Clinical and Community Interventions. 2. Thousand Oaks (CA): Sage Publications, Inc., 1991.

\section{Krug 2002}

Krug EG, Dahlberg LL, Mercy JA, Zwi AB, Lozano R, editor(s). World Report on Violence and Health. Geneva $(\mathrm{CH})$ : World Health Organization, 2002.

\section{Kubany 1996}

Kubany ES, Haynes SN, Abueg FR, Manke FP, Brennan JM, Stahura C. Development and validation of the Trauma-Related Guilt Inventory (TRGI). Psychological Assessment 1996;8(4):42844. [DOI: 10.1037/1040-3590.8.4.428]

\section{Kulka 1988}

Kulka RA, Schlenger WE, Fairbank JA, Hough RL, Jordan BK, Marmar CR, et al. National Vietnam Veterans Readjustment Study (NVVRS): Description, Current Status, and Initial PTSD Prevalence Estimates. Final Report. Washington (DC): Veterans Administration, 1988

\section{Lerman 2019}

Lerman I, Davis B, Huang M, Huang C, Sorkin L, Proudfoot J, et al. Noninvasive vagus nerve stimulation alters neural response and physiological autonomic tone to noxious thermal challenge. PlOS One 2019;14(2):e0201212. [DOI: 10.1371/ journal.pone.0201212; PMC6373934; PUBMED: 30759089]

\section{Liu 2017}

Liu H, Petukhova MV, Sampson NA, Aguilar-Gaxiola S, Alonso J, Andrade LH, et al. Association of DSM-IV post-traumatic stress disorder with traumatic experience type and history in the World Health Organization World Mental Health Surveys. JAMA Psychiatry 2017;74(3):270-81. [DOI: 10.1001/ jamapsychiatry.2016.3783; PMC5441566 ; PUBMED: 28055082]

\section{Lonsway 1994}

Lonsway KA, Fitzgerald LF. Rape myths: in review. Psychology of Women Quarterly 1994;18(2):133-64. [DOI: 10.1111/

j.1471-6402.1994.tb00448.x]

\section{MacDonald 2012}

MacDonald G, Higgins JPT, Ramchandani P, Valentine JC, Bronger LP, Klein P, et al. Cognitive-behavioural interventions for children who have been sexually abused. Cochrane Database of Systematic Reviews 2012, Issue 5. [DOI: 10.1002/14651858.CD001930.pub3; PUBMED: 22592679]

\section{MacMillan 2009}

Macmillan HL, Wathen CN, Barlow J, Fergusson DM, Leventhal JM, Taussig HN. Interventions to prevent child maltreatment and associated impairment. Lancet 2009;373(9659):250-66. [DOI: 10.1016/S0140-6736(08)61708-0]

\section{Marsella 1996}

Marsella AJ, Friedman MJ, Spain EH. Ethnocultural aspects of PTSD: an overview of issues and research directions. In: Marsella AJ, Friedman MJ, Gerrity ET, Scurfield RM editor(s). Ethnocultural Aspects of Posttraumatic Stress Disorder: Issues, Research, and Clinical Applications. Vol. 12, Washington (DC): American Psychological Association, 1996:105-29.

\section{McLellan 1980}

McLellan AT, Luborsky L, Woody GE, O'Brien CP. An improved diagnostic evaluation instrument for substance abuse patients. The Addiction Severity Index. Journal of Nervous and Mental Disease 1980;168(1):26-33. [PUBMED: 7351540]

\section{McLellan 1992}

McLellan AT, Kushner H, Metzger D, Peters R, Smith I, Grissom G, et al. The Fifth Edition of the Addiction Severity Index. Journal of Substance Abuse Treatment 1992;9(3):199-213. [PUBMED: 1334156]

\section{Meichenbaum 1977}

Meichenbaum D. Cognitive-Behavior Modification: An Integrative Approach. New York (NY): Plenum Press, 1977. [ISBN 978-1-4757-9739-8]

\section{Miller 2015}

Miller KE, Cranston CC, Davis JL, Newman E, Resnick H. Psychological outcomes after a sexual assault video intervention: a randomized trial. Journal of Forensic Nursing 2015;11(3):129-36. [DOI: 10.1097/JFN.0000000000000080; PUBMED: 26291847]

\section{Moher 2009}

Moher D, Liberati A, Tetzlaff J, Altman DG, PRISMA Group. Preferred reporting items for systematic reviews and meta-analyses: the PRISMA statement. PLOS Medicine 2009;6(7):e1000097. [DOI: 10.1371/journal.pmed.1000097; PMC2707599; PUBMED: 19621072]

\section{Morgan 2017}

Morgan RE, Kena G. Criminal victimization, 2016. www.bjs.gov/ content/pub/pdf/cv16_old.pdf (accessed 3 January 2019). 


\section{Neville 1999}

Neville HA, Heppner MJ. Contextualizing rape: reviewing sequelae and proposing a culturally inclusive ecological model of sexual assault recovery. Applied \& Preventative Psychology 1999;8(1):41-62. [DOI: 10.1016/S0962-1849(99)80010-9]

\section{NHS 2015}

NHS England. Commissioning framework for adult and paediatric Sexual Assault Referral Centres (SARC) services. www.england.nhs.uk/commissioning/wp-content/uploads/ sites/12/2013/05/SARCs-service-spec-contract-template-andpaed-framework.pdf (accessed 3 January 2019).

\section{NICE 2018}

National Institute for Health and Care Excellence. Posttraumatic stress disorder. www.nice.org.uk/guidance/ng116 (accessed 3 January 2019).

\section{NSVRC 2011}

National Sexual Violence Resource Center. Assessing patients for sexual violence: a guide for health care providers. www.nsvrc.org/sites/default/files/ Publications_NSVRC_Guides_Assessing-patients-for-sexualviolence.pdf (accessed 3 January 2019).

\section{ONS 2015}

Office for National Statistics. Violent Crime and Sexual Offences - Intimate Personal Violence and Serious Sexual Assault. Findings from the 2013/14 Crime Survey for England and Wales and Police Recorded Crime Over the Same Period on Violent Crime and Sexual Offences. London (UK): Office for National Statistics, 2015.

\section{Parcesepe 2015}

Parcesepe AM, Martin SL, Pollock MD, Garcia-Moreno C. The effectiveness of mental health interventions for adult female survivors of sexual assault: a systematic review. Aggression and Violent Behavior 2015;25(Part A):15-25. [DOI: 10.1016/ j.avb.2015.06.004]

\section{Parmar 1998}

Parmar MK, Torri V, Stewart LA. Extracting summary statistics to perform meta-analyses of the published literature for survival endpoints. Statistics in Medicine 1998;17(24):2815-34. [PUBMED: 9921604]

\section{Peterson 2017}

Peterson C, DeGue S, Florence C, Lokey CN. Lifetime economic burden of rape among US adults. American Journal of Preventive Medicine 2017;52(6):691-701. [DOI: 10.1016/ j.amepre.2016.11.014; PMC5438753 ; PUBMED: 28153649]

\section{Pietrzak 2011}

Pietrzak RH, Goldstein RB, Southwick SM, Grant BF. Prevalence and Axis I comorbidity of full and partial posttraumatic stress disorder in the United States: results from Wave 2 of the National Epidemiologic Survey on Alcohol and Related Conditions. Journal of Anxiety Disorders 2011;25(3):456-65. [DOI: 10.1016/j.janxdis.2010.11.010; PMC3051041; PUBMED: 21168991]

\section{Pitman 1996}

Pitman RK, Orr SP, Altman B, Longpre RE, Poiré RE, Macklin ML. Emotional processing during eye movement desensitization and reprocessing therapy of Vietnam veterans with chronic posttraumatic stress disorder. Comprehensive Psychiatry 1996;37(6):419-29. [PUBMED: 8932966]

\section{Pocock 1987}

Pocock SJ, Hughes MD, Lee RJ. Statistical problems in the reporting of clinical trials. A survey of three medical journals. New England Journal of Medicine 1987;317(7):426-32. [DOI: 10.1056/NEJM198708133170706; PUBMED: 3614286]

\section{Pradhan 2012}

Pradhan B, Chappuis F, Baral D, Karki P, Rijal S, Hadengue A, et al. The Alcohol Use Disorders Identification Test (AUDIT): validation of a Nepali version for the detection of alcohol use disorders and hazardous drinking in medical settings. Substance Abuse Treatment, Prevention and Policy 2012;7:42. [DOI: 10.1186/1747-597X-7-42; PMC3508982; PUBMED: 23039711]

\section{Radloff 1977}

Radloff LS. The CES-D scale: a self-report depression scale for research in the general population. Applied Psychological Measurement 1977;1(3):385-401. [DOI: 10.1177/014662167700100306]

\section{Regehr 2013}

Regehr C, Alaggia R, Dennis J, Pitts A, Saini M. Interventions to reduce distress in adult victims of rape and sexual violence: a systematic review. Research on Social Work Practice. 2013;23(3):257-65. [DOI: 10.1177/1049731512474103]

\section{Resick 1988}

Resick PA, Jordan CG, Girelli SA, Hutter CK, Marhoefer-Dvorak S. A comparative outcome study of behavioral group therapy for sexual assault victims. Behavior Therapy 1988;19(3):385-401. [DOI: 10.1016/S0005-7894(88)80011-X]

\section{Resick 1992}

Resick PA, Schnicke MK. Cognitive processing therapy for sexual assault victims. Journal of Consulting and Clinical Psychology 1992;60(5):748-56. [PUBMED: 1401390]

\section{Resick 1993}

Resick PA, Schnicke MK. Cognitive Processing Therapy for Sexual Assault Victims: A Treatment Manual. Newbury Park (CA): Sage Publications Inc., 1993.

\section{Resnick 1977}

Resnick HS, Yehuda R, Acierno R. Acute post-rape plasma cortisol, alcohol use, and PTSD symptom profile among recent rape victims. Annals of the New York Academy of Sciences 1997;821(1):433-6. [PUBMED: 9238223]

\section{Review Manager 2014 [Computer program]}

Nordic Cochrane Centre, The Cochrane Collaboration. Review Manager 5 (RevMan 5). Version 5.3. Copenhagen: Nordic Cochrane Centre, The Cochrane Collaboration, 2014. 


\section{Review Manager Web 2019 [Computer program]}

The Cochrane Collaboration. Review Manager Web (RevMan Web). The Cochrane Collaboration, 2019.

\section{Roberts 2015}

Roberts NP, Roberts PA, Jones N, Bisson JI. Psychological interventions for post-traumatic stress disorder and comorbid substance use disorder: a systematic review and meta-analysis. Clinical Psychology Review 2015;38:25-38. [DOI: 10.1016/ j.cpr.2015.02.007; PUBMED: 25792193]

\section{Rose 2002}

Rose S, Bisson J, Churchill R, Wessely S. Psychological debriefing for preventing post traumatic stress disorder (PTSD). Cochrane Database of Systematic Reviews 2002, Issue 2. [DOI: 10.1002/14651858.CD000560; PUBMED: 12076399]

\section{Rothbaum 1990}

Rothbaum BO, Dancu CV, Riggs D, Foa EB. The PTSD Symptom Scale. European Association of Behavior Therapy Convention; 1990 Sep 12-15; Paris (FR). 1990.

\section{Rothbaum 1997}

Rothbaum BO. A controlled study of eye movement desensitization and reprocessing in the treatment of posttraumatic stress disordered sexual assault victims. Bulletin of the Menninger Clinic 1997;61(3):317-34. [PUBMED: 9260344]

\section{Rozental 2018}

Rozental A, Castonguay L, Dimidjian S, Lambert M, Shafran R, Andersson G, et al. Negative effects in psychotherapy: commentary and recommendations for future research and clinical practice. British Journal of Psychiatry Open 2018;4(4):307-12. [DOI: 10.1192/bjo.2018.42; PMC6066991; PUBMED: 30083384]

\section{Sandy 1998}

Sandy PR. The socio-cultural context of rape: a crosscultural study. Journal of Social Issues 1981;37(4):5-27. [DOI: 10.1111/j.1540-4560.1981.tb01068.x; psycnet.apa.org/ record/1982-10058-001]

\section{Santa Mina 2006}

Santa Mina EE, Gallop R, Links P, Heslegrave R, Pringle D, Wekerle C, et al. The Self-Injury Questionnaire: evaluation of the psychometric properties in a clinical population. Journal of Psychiatric and Mental Health Nursing 2006;13(2):221-7. [DOI: 10.1111/j.1365-2850.2006.00944.x; PUBMED: 16608478]

\section{Schünemann 2011}

Schünemann HJ, Oxman AD, Vist GE, Higgins JPT, Deeks JJ, Glasziou P, et al. Cochrane Applicability and Recommendations Methods Group. Chapter 12: Interpreting results and drawing conclusions. In: Higgins JP, Green S, editor(s). Cochrane Handbook for Systematic Reviews of Interventions Version 5.1.0 (updated March 2011). The Cochrane Collaboration, 2011. Available from handbook.cochrane.org.

\section{Schünemann 2017}

Schünemann HJ, Oxman AD, Higgins JPT, Vist GE, Glasziou P, Akl E, et al. Cochrane GRADEing Methods Group and the
Cochrane Statisticial Methods Group. Chapter 11: Completing 'Summary of findings' tables and grading the confidence in or quality of the evidence. In: Higgins JP, Churchill R, Chandler J, Cumpston MS, editor(s). Cochrane Handbook for Systematic Reviews of Interventions version 5.2.0 (updated June 2017). The Cochrane Collaboration, 2017. Available from www.training.cochrane.org/handbook.

\section{Selzer 1971}

Selzer ML. The Michigan Alcoholism Screening Test: the quest for a new diagnostic instrument. American Journal of Psychiatry 1971;127(12):1653-8. [DOI: 10.1176/ajp.127.12.1653; PUBMED: $5565851]$

\section{Shapiro 1995}

Shapiro F. Eye Movement Desensitization and Reprocessing: Basic Principles, Protocols, and Procedures. New York (NY): Guilford Press, 1995.

\section{Sherman 1998}

Sherman JJ. Effects of psychotherapeutic treatments for PTSD: a meta-analysis of controlled clinical trials. Journal of Traumatic Stress 1998;11(3):413-35. [DOI: 10.1023/A:1024444410595; PUBMED: 9690185 ]

\section{Sikkema 2018}

Sikkema KJ, Choi KW, Robertson C, Knettel BA, Ciya N, Knippler ET, et al. Development of a coping intervention to improve traumatic stress and HIV care engagement among South African women with sexual trauma histories. Evaluation and Program Planning 2018;68:148-56. [DOI: org/10.1016/ j.evalprogplan.2018.02.007; PMC5953816; PUBMED: 29597104]

\section{Singer 2003}

Singer M, Clair S. Syndemics and public health: reconceptualizing disease in bio-social context. Medical Anthropology Quarterly 2003;17(4):423-41. [PUBMED: 14716917]

\section{Singh 2011}

Singh D, Chaudoir SR, Escobar MC, Kalichman S. Stigma, burden, social support, and willingness to care among caregivers of PLWHA in home-based care in South Africa. AIDS Care 2011;23(7):839-45. [DOI: 10.1080/09540121.2010.542122; PMC3125468; PUBMED: 21400316]

\section{Skevington 2004}

Skevington SM, Lotfy M, O'Connell KA, WHOQOL Group. The World Health Organization's WHOQOL-BREF quality of life assessment: psychometric properties and results of the international field trial. A report from the WHOQOL group. Quality of Life Research 2004;13(2):299-310. [DOI: 10.1023/ B:QURE.0000018486.91360.00; PUBMED: 15085902]

\section{Skinner 1982}

Skinner H. The Drug Abuse Screening Test. Addictive Behaviors 1982;7(4):363-71. [PUBMED: 7183189]

\section{Spielberger 1970}

Spielberger CD, Gorsuch RL, Lushene RE. STAI Manual for the State-Trait Anxiety Inventory. Palo Alto (CA): Consulting Psychologists Press, 1970. [hdl.handle.net/10477/2895] 


\section{Spitzer 1999}

Spitzer RL, Kroenke K, Williams JB. Validation and utility of a self-report version of PRIME-MD. The PHQ Primary Care Study. JAMA 1999;282(18):1737-44. [DOI: 10.1001/jama.282.18.1737]

\section{Spitzer 2006}

Spitzer RL, Kroenke K, Williams JB, Löwe B. A brief measure for assessing generalized anxiety disorder: the GAD-7. Archives of Internal Medicine 2006;166(10):1092-7. [DOI: 10.1001/ archinte.166.10.1092; PUBMED: 16717171]

\section{Stata 2017 [Computer program]}

StataCorp. Stata. Version 15. College Station, TX, USA: StataCorp, 2017.

\section{Sterne 2019}

Sterne JAC, Savović J, Page MJ, Elbers RG, Blencowe NS, Boutron I, et al. RoB 2: a revised tool for assessing risk of bias in randomised trials. BMJ 2019;366:14898. [DOI: 10.1136/ bmj.14898; PUBMED: 31462531]

\section{Tan 2018}

Tan M, O'Doherty L, Gilchrist G, Taft A, Feder G, Tirado Muñoz J, et al. Psychological therapies for women who experience intimate partner violence. Cochrane Database of Systematic Reviews 2018, Issue 5. [DOI: 10.1002/14651858.CD013017]

\section{Tannock 1996}

Tannock IF. False-positive results in clinical trials: multiple significance tests and the problem of unreported comparisons. Journal of the National Cancer Institute 1996;88(3-4):206-7. [DOI: 10.1093/jnci/88.3-4.206; PUBMED: 8632495]

\section{Tewkesbury 2007}

Tewkesbury R. Effects of sexual assaults on men: physical, mental and sexual consequences. International Journal of Men's Health 2007;6(1):22-35. [DOI: 10.3149/jmh.0601.22]

\section{Tierney 2007}

Tierney JF, Stewart LA, Ghersi D, Burdett S, Sydes MR. Practical methods for incorporating summary time-to-event data into meta-analysis. Trials 2007;8:16. [DOI: 10.1186/1745-6215-8-16; PMC1920534; PUBMED: 17555582]

\section{Trabold 2018}

Trabold N, McMahon J, Alsobrooks S, Whitney S, Mittal M. A systematic review of intimate partner violence interventions: state of the field and implications for practitioners. Trauma, Violence, \& Abuse 2018 Jan 1 [Epub ahead of print]. [DOI: 10.1177/1524838018767934; PUBMED: 29649966]

\section{Vandenberghe 2018}

Vandenberghe A, Hendriks B, Peeters L, Roelens K, Keygnaert I. Establishing sexual assault care centres in Belgium: health professionals' role in the patient-centred care for victims of sexual violence. BMC Health Services Research 2018;18(1):807. [DOI: 10.1186/s12913-018-3608-6; PMC6196455; PUBMED: 30348151]

\section{Vaux 1986}

Vaux A, Phillips J, Holly L, Thomson B, Williams D, Stewart D. The Social Support Appraisals (SS-A) Scale: studies of reliability and validity. American Journal of Community Psychology 1986;14(2):195-219. [DOI: 10.1007/BF00911821]

\section{Veronen 1983}

Veronen LJ, Kilpatrick DG. Stress management for rape victims. In: Meichenbaum D, Jaremko ME, editor(s) editor(s). Stress Reduction and Prevention. New York (NY): Plenum Press, 1983:341-74.

\section{Vickerman 2009}

Vickerman KA, Margolin G. Rape treatment outcome research: empirical findings and state of the literature. Clinical Psychology Review 2009;29(5):431-48. [DOI: 10.1016/j.cpr.2009.04.004; PMC2773678; PUBMED: 19442425]

\section{Walby 2016}

Walby S, Towers J, Francis B. Is violent crime increasing or decreasing? A new methodology to measure repeat attacks making visible the significance of gender and domestic relations. British Journal of Criminology 2016;56(6):1203-34. [DOI: 10.1093/bjc/azv131]

\section{Walker 2005}

Walker J, Archer J, Davies M. Effects of rape on men: a descriptive analysis. Archives of Sexual Behavior 2005;34(1):69-80. [DOI: 10.1007/s10508-005-1001-0; PUBMED: 15772770]

\section{Walters 2013}

Walters ML, Chen J, Breiding MJ. The National Intimate Partner and Sexual Violence Survey (NISVS): 2010 Findings on Victimization by Sexual Orientation. Atlanta (GA): National Center for Injury Prevention and Control, Centers for Disease Control and Prevention, 2013.

\section{Wasco 2003}

Wasco SM. Conceptualizing the harm done by rape: applications of trauma theory to experiences of sexual assault. Trauma, Violence \& Abuse 2003;4(4):309-22. [DOI: 10.1177/1524838003256560]

\section{Weiss 1997}

Weiss DS, Marmar CR. The Impact of Event Scale - Revised. In: Wilson JP, Keane TM editor(s). Assessing Psychological Trauma and PTSD. New York (NY): Guilford Press, 1997:399-411. [pp. 399-411]

\section{WHO 2002}

Krug EG, Dahlberg LL, Mercy JA, Zwi AB, Lozano R. World Report on Violence and Health. Geneva $(\mathrm{CH})$ : World Health Organization, 2002.

\section{WHO 2005}

García-Moreno C, Jansen HAFM, Ellsberg M, Heise L, Watts C. WHO Multi-Country Study on Women's Health and Domestic Violence Against Women: Initial Results on Prevalence, Health Outcomes and Women's Responses. Geneva $(\mathrm{CH})$ : World Health Organization, 2005. 


\section{WHO 2013a}

World Health Organization. Global and Regional Estimates of Violence Against Women: Prevalence and Health Effects of Intimate Partner Violence and Non-Partner Sexual Violence. Geneva (CH): World Health Organization, 2013.

\section{WHO 2013b}

World Health Organization. Responding to Intimate Partner Violence and Sexual Violence Against Women: WHO Clinical and Policy Guidelines. Geneva ( $\mathrm{CH})$ : World Health Organization, 2013.

\section{WHO/PAHO 2012}

World Health Organization, Pan American Health Organization. Understanding and addressing violence against women: human trafficking. apps.who.int/iris/bitstream/handle/10665/77394/ WHO_RHR_12.42_eng.pdf?sequence=1 (accessed prior to 9 July 2019).

\section{A P P E N D I CES}

\section{Appendix 1. How the intervention might work}

Cognitive-behavioural interventions are based on the proposition that behaviours are cognitively mediated (Butler 2006). Mental health and social problems may be influenced by cognitions and resulting behaviours. Because cognitive activity may be monitored and altered, behaviours may be changed through cognitive changes (Dobson 2009). Therefore, addressing certain thinking patterns and beliefs may result in positive changes in symptoms, problems and behaviours, which may reduce some of the negative consequences of rape or sexual assault (Butler 2006). In the case of trauma, theorists believe that the appraisal of fear involves the activation of trauma-induced schema that lead the survivor to pay attention to information that is consistent with the schema and to ignore evidence that is inconsistent (Resick 1992). This means that benign or ambiguous events can trigger a fear appraisal in trauma survivors (Beck 1985). Hence, cognitive theory, as applied to the process of PTSD (Veronen 1983), focusses on two processes: (1) changing a person's cognitive appraisal of the traumatic event, or changing the process by which an individual attaches meaning to an event; and (2) changing a person's attribution of the event. Coping skill treatments are designed to equip victims with an array of skills to manage their trauma. Some interventions are designed to be delivered within a short period of time following the assault or rape (e.g. less than three months), whereas others are used for survivors over the longer term. The former attempt to provide prophylactic treatment to prevent chronic problems, while others intend to facilitate faster recovery (Vickerman 2009).

Interventions for sexual assault and rape survivors typically employ a combination of approaches; for example, in the case of SIT, PET, and CPT, as outlined below.

SIT was adapted by Veronen and Kilpatrick (Veronen 1983) from anxiety management procedures (Meichenbaum 1977). It incorporates three elements: (1) behaviourally-based psycho-education so that victims can understand and normalise fear and avoidance behaviours; (2) guided hierarchical in vivo assignments to target rape-related phobias (e.g. darkness); and (3) training in six behavioural and cognitive-behavioural coping strategies, which are thought-stopping, guided self-dialogue, muscle relaxation, controlled breathing, covert modelling, and role playing. The goal of SIT is to increase the survivor's awareness of conditioned stimuli to improve early detection of anxiety-provoking cues, which facilitates the use of coping skills early in the stress response to reduce anxiety (Sherman 1998).

PET was developed from earlier treatments using flooding exposure techniques and emotion processing theory with anxiety disordered patients (Foa 1986). These techniques were extended by Foa and colleagues (Foa 1986; Foa 1994), who argued that it is the encoding of memories under extreme distress that leads to disjointed and disorganised memories, which impede natural recovery and lead to PTSD. The aim of PET is to decrease the anxiety associated with rape memories. PET begins with psycho-education, breathing training, and the development of a fear and avoidance hierarchy for in vivo exposures. In therapy, victims are asked to relive the rape scene and describe it aloud as they are imagining it, using present tense and vivid detail, which may be done several times in one therapy session. The victim's narrative is recorded and daily homework requires the victim to listen to their recorded account for further exposure (Foa 1991).

CPT was developed from emotional processing theory to identify a rape victim's 'stuck points', which are the parts of the traumatic narratives that cause them the greatest conflict (Resick 1992; Resick 1993). These are manifestations of unsuccessful attempts to accommodate information in relation to the trauma into pre-existing memory and belief structures. The goal of CPT is to help victims to integrate their trauma into pre-existing schemas, to decrease avoidance and intrusions of unintegrated aspects of the trauma. Unlike PET, CPT seeks to directly correct participants' misconceptions or misinformation about their trauma (for example, 'I'm not safe anywhere' or 'I can't trust anyone'). CPT also includes psycho-education, exposure and cognitive methods. Exposure is achieved via the victim writing accounts of the rape and its meaning, which the victim rereads between sessions and writes about the impact of the trauma multiple times, in order

Psychosocial interventions for survivors of rape and sexual assault experienced during adulthood (Protocol) 
to incorporate new understandings and evaluation. Therapy then addresses one of five themes (safety, trust, power/control, esteem or intimacy) in each of the last five sessions, via the use of cognitive-restructuring worksheets, Socratic questioning and discussion.

Behavioural therapies are based on the premise that all behaviours are learned and, therefore, that unhealthy behaviours can be changed. Techniques such as systematic desensitisation and flooding are often used with this population, which emphasise the importance of extinguishing anxiety and reducing avoidant behaviours. For example, Foa and colleagues believe that exposure to the trauma allows mistaken evaluations and faulty stimulus-response associations to be corrected (Foa 1986; Foa 1994). Victims are taught to replace a fear response with relaxation responses. This can be done gradually, with systematic desensitisation, or more quickly via flooding.

EMDR was developed by Shapiro 1995 for the treatment of PTSD. It involves exposure elements and cognitive techniques. In EMDR, a scene is used to represent the entire rape trauma. The survivor imagines the scene and recites words related to the scene, while the therapist moves his or her fingers back and forth in front of the survivor, so that the survivor performs rhythmic, multi-saccadic eye movements (quick, simultaneous movements of both eyes between two or more phases of fixation in the same direction) by watching the therapist's fingers. This movement is argued to facilitate the processing of trauma memory through the dual attention required to focus on attending to the therapist's finger movement (external stimulus) and the trauma scene (internal stimulus). When the survivor's anxiety to the scene has decreased, a new adaptive belief is rehearsed until this new belief feels true (Rothbaum 1997). EMDR is similar to the behavioural techniques of flooding and systematic desensitisation (Boudewyns 1996a), and studies comparing EMDR with and without eye movements show that EMDR without eye movements leads to equivalent outcomes as EDMR with eye movements (Boudewyns 1996b; Pitman 1996). EMDR is thought to work for patients who have been traumatised by the fact that eye movements can reduce the intensity of disturbing thoughts under certain conditions (Bisson 2013).

Third wave CBTs (e.g. acceptance and commitment therapy and mindfulness) act on changing the function of psychological events and the individuals relationship to them through acceptance, being present, and committed action (Hayes 2006).

Counselling encompasses a range of interventions that may be employed by, for example, rape crisis centres (Cryer 1980; Foa 1991; Resick 1988). Counselling can be premised on a number of approaches (e.g. humanist, psychodynamic) and may be delivered as an intervention in itself or in combination with other approaches. Counselling is likely to be very individually focused in order to discuss issues raised by the survivor, and the necessary variation makes it difficult to understand exactly what is included in each session.

Humanistic and supportive therapies include an eclectic mix of therapeutic techniques. Supportive therapy is almost always non-directive, that is, the survivor is empowered to guide the content and the therapist avoids offering direct advice (Cohen 2005; Deblinger 2001). The focus is on developing a supportive, emotionally-involved relationship between the therapist and participant (Cohen 2005). Supportive therapy can be conducted in either an individual or group format.

Other psychologically-orientated interventions include a diverse range of therapies that aim to help survivors cope with, express and work through trauma via, for example, expressive writing (Harte 2013) or mindfulness (Brotto 2012). For instance, equine-assisted therapy for anxiety and post-traumatic stress symptoms has been shown to reduce symptoms of post-traumatic stress, severe emotional responses to trauma, generalised anxiety, symptoms of depression and alcohol use, as well as increasing the use of mindfulness strategies (Earles 2015).

Psychosocial interventions include a wide range of interventions that target interpersonal, social and environmental factors that relate to recovery from the trauma of rape and sexual assault in addition to, or instead of, the individual factors that are the focus of psychological therapies. The way in which the interventions might work will be dependent on the factors that are targeted. Psycho-education elements aim to provide information, modelling and training, for example, to explain maladaptive and adaptive coping strategies and to encourage the use of the latter (e.g. see Sikkema 2018). Group programmes and the provision of advisors or mentors provide social support, which can be important given the stigma and shame associated with rape and sexual assault that can lead to social isolation. These can increase self-esteem (Sikkema 2018), and provide practical assistance and emotional support (Home Office 2017).

\section{Appendix 2. MEDLINE search strategy}

1 sex offenses/

2 Incest/

3 intimate partner violence/

4 human trafficking/

5 rape/

6 spouse abuse/

7 (sex\$ adj5 (abuse $\$$ or assaul\$ or attack\$ or aggress $\$$ or coer $\$$ or exploit $\$$ or force $\$$ or molest $\$$ or offen $\$$ or traffick\$ or trauma $\$$ or unlawful\$ or unwanted or violen\$)).tw,kf.

8 (intercourse adj5 (coer\$ or force\$ or unwanted)).tw,kf.

9 intimate partner violence.tw,kf.

10 (rape or raped or incest\$).tw,kf.

11 (sex\$ adj3 (victim\$ or revictim\$ or re-victim\$ or survivor\$)).tw,kf.

12 or/1-11

13 Anxiety/th

Psychosocial interventions for survivors of rape and sexual assault experienced during adulthood (Protocol)

Copyright (c) 2019 The Cochrane Collaboration. Published by John Wiley \& Sons, Ltd. 
14 Anxiety Disorders/th

15 Adaptation, Psychological/

16 exp Behavior Therapy/

17 Combined Modality Therapy/

18 community networks/

19 exp Complementary therapies/

20 exp Counseling/

21 Depression/th

22 Depressive Disorder/th

23 Depressive Disorder, Major/th

24 Exercise/

25 Exercise therapy/

26 Health Education/

27 Health Knowledge, Attitudes, Practice/

28 Interview, Psychological/

29 exp mind body therapies/

30 Psychological adjustment/

31 Psychological Trauma/pc, rh, th

32 psychosocial support systems/

33 exp psychotherapy/

34 "Referral and Consultation"/

35 Self-Help Groups/ (8831)

36 Social Support/

37 Stress Disorders, Post-Traumatic/pc, rh, th

38 video recording/ or videotape recording/

39 Writing/

40 ((abreaction or desensitization or exposure or implosive) adj3 therap\$).tw,kf.

41 "acceptance and commitment therapy".tw,kf.

42 (advisor\$ or advocate\$ or advocacy).tw,kf.

43 ((animal\$ or art or colo?r or creative or dance or dancing or drama or equine or experiential or music or narrative or play\$ or sensory or singing) adj3 (program\$ or intervention\$ or therap\$)).tw,kf.

44 (autogenic or autosuggestion\$ or auto-suggestion\$ or breathing exercise $\$$ or hypnosis or hypno-therapy or hypnotherapy).tw,kf.

45 behavio $\$$ activation.tw,kf.

46 (behavio?r\$ adj3 (intervention\$ or program\$ or therap\$ or training or treatment\$)).tw,kf.

47 ((biofeedback or feedback or imagery) adj3 (intervention\$ or therap\$ or train $\$$ or treatment\$)).tw,kf.

48 ((brief or combination or compass\$ focus\$ or integrated or integrative or time-limited) adj3 (intervention\$ or therap\$ or treatment\$)).tw,kf.

49 ((client focus\$ or non-direct\$ or nondirect\$ or solution focus\$ or trauma\$ or talking) adj3 therap\$).tw,kf.

50 (cognitiv\$ or cognition).tw,kf.

51 CBT.tw,kf.

52 ((cope or coping) adj1 (intervention\$ or mechanism\$ or skill\$ or technique\$)).tw,kf.

53 counsel?ing.tw,kf.

54 ((couple\$ or family or group or systemic $\$$ or multimodal\$ or multi-modal\$) adj3 (program\$ or intervention\$ or therap\$ or treat\$)).tw,kf. 55 dialectical behavio? $\$$ therap\$.tw,kf.

56 (exercise\$ or physical training).tw,kf.

57 ((existential or gestalt or humanistic or interpersonal or milieu or person-centred or residential or socioenvironmental or socio-environmental) adj1 therap\$).tw.kf.

58 expressive writing.tw,kf.

59 ("Eye Movement Desensitization and Reprocessing" or EMDR).tw,kf. (439)

60 (meditat\$ or mental training or mindfulness\$ or mind training or brain training or yoga).tw,kf.

61 motivational interview\$.tw,kf.

62 (reality therap\$ or problem solving).tw,kf.

63 (psycho\$ therap\$ or psychotherap\$).tw,kf.

64 (psychoanalytic\$ or psycho-analytic\$ or psychodynamic\$ or psycho-dynamic\$).tw,kf.

65 (psychodrama or psycho-drama or acting out or role play).tw,kf.

66 (psychosocial or psycho-social or psychoeducation\$ or psycho-education\$).tw,kf.

67 rational emotive.tw,kf.

68 (Relax\$ adj3 (training\$ or treatment\$ or therap\$)).tw,kf.

69 (Service\$ adj3 (refer\$ or use\$)).tw,kf.

70 (stress inoculation training or SIT or prolonged exposure therapy or PET or cognitive processing therapy or CPT).tw,kf.

71 ((support or advice or advis $\$ 1$ ) adj 1 (centre $\$ 1$ or center $\$ 1$ or community or group\$ or network\$ or social or staff\$)).tw,kf. 72 (therapeutic allianc\$ or therapeutic relationship\$ or therapeutic communit\$).tw,kf.

Psychosocial interventions for survivors of rape and sexual assault experienced during adulthood (Protocol) 
73 Third wave.tw,kf.

74 or/13-73

7512 and 74

76 (rape adj3 (centre\$ or center\$ or service\$ or support)).tw,kf.

77 ((sex\$ assault adj3 centre) or (sex\$ assault adj3 center) or (sex\$ assault adj3 service) or (sex\$ assault adj3 support)).tw,kf.

78 ((sex\$ abuse $\$$ adj3 centre) or (sex\$ abuse $\$$ adj3 center) or (sex\$ abuse $\$$ adj3 service) or (sex $\$$ abuse $\$$ adj3 support)).tw,kf.

79 or/76-78

8075 or 79

81 randomized controlled trial.pt.

82 controlled clinical trial.pt.

83 randomi\#ed.ab.

84 placebo\$.ab.

85 drug therapy.fs.

86 randomly.ab.

87 trial.ab.

88 groups.ab.

89 or/ $/ 81-88$

90 exp animals/ not humans.sh.

9189 not 90

9280 and 91

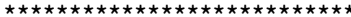

\section{Appendix 3. Cochrane Common Mental Disorders Controlled Trials Register}

\section{Core MEDLINE search}

The search strategy below is the weekly OVID Medline search, which was used to inform the Group's specialised register. It is based on a list of terms for all conditions within the scope of the Cochrane Common Mental Disorders Group plus a sensitive RCT filter.

\section{1. [MeSH Headings]:}

eating disorders/ or anorexia nervosa/ or binge-eating disorder/ or bulimia nervosa/ or female athlete triad syndrome/ or pica/ or hyperphagia/ or bulimia/ or self-injurious behavior/ or self mutilation/ or suicide/ or suicidal ideation/ or suicide, attempted/or mood disorders/ or affective disorders, psychotic/ or bipolar disorder/ or cyclothymic disorder/ or depressive disorder/ or depression, postpartum/ or depressive disorder, major/ or depressive disorder, treatment-resistant/ or dysthymic disorder/ or seasonal affective disorder/ or neurotic disorders/ or depression/ or adjustment disorders/ or exp antidepressive agents/ or anxiety disorders/ or agoraphobia/ or neurocirculatory asthenia/ or obsessive-compulsive disorder/ or obsessive hoarding/ or panic disorder/ or phobic disorders/or stress disorders, traumatic/ or combat disorders/ or stress disorders, post-traumatic/ or stress disorders, traumatic, acute/ or anxiety/ or anxiety, castration/ or koro/ or anxiety, separation/ or panic/ or exp anti-anxiety agents/or somatoform disorders/or body dysmorphic disorders/or conversion disorder/ or hypochondriasis/ or neurasthenia/ or hysteria/ or munchausen syndrome by proxy/or munchausen syndrome/ or fatigue syndrome, chronic/ or obsessive behavior/ or compulsive behavior/ or behavior, addictive/ or impulse control disorders/ or firesetting behavior/ or gambling/ or trichotillomania/ or stress, psychological/ or burnout, professional/ or sexual dysfunctions, psychological/ or vaginismus/ or Anhedonia/ or Affective Symptoms/ or * Mental Disorders/

\section{2. [Title/ Author Keywords]:}

(eating disorder ${ }^{\star}$ or anorexia nervosa or bulimi* or binge eat ${ }^{\star}$ or (self adj (injur ${ }^{\star}$ or mutilat ${ }^{\star}$ )) or suicide ${ }^{\star}$ or suicidal or parasuicid ${ }^{\star}$ or mood disorder $^{\star}$ or affective disorder ${ }^{\star}$ or bipolar i or bipolar ii or (bipolar and (affective or disorder $\left.{ }^{\star}\right)$ ) or mania or manic or cyclothymic ${ }^{\star}$ or depression or depressive or dysthymi ${ }^{\star}$ or neurotic or neurosis or adjustment disorder* or antidepress* or anxiety disorder ${ }^{\star}$ or agoraphobia or obsess ${ }^{\star}$ or compulsi ${ }^{\star}$ or panic or phobi ${ }^{\star}$ or ptsd or posttrauma* or post trauma* or combat or somatoform or somati\#ation or medical ${ }^{\star}$ unexplained or body dysmorphi* or conversion disorder or hypochondria* or neurastheni* or hysteria or munchausen or chronic fatigue ${ }^{\star}$ or gambling or trichotillomania or vaginismus or anhedoni* or affective symptoms or mental disorder* or mental health).ti,kf.

\section{3. [RCT filter]:}

(controlled clinical trial.pt. or randomised controlled trial.pt. or (randomi\#ed or randomi\#ation).ab,ti. or randomly.ab. or (random* adj3 (administ ${ }^{\star}$ or allocat ${ }^{\star}$ or assign* or class ${ }^{\star}$ or control ${ }^{\star}$ or determine* or divide* or distribut ${ }^{\star}$ or expose ${ }^{\star}$ or fashion or number ${ }^{\star}$ or place $^{\star}$ or recruit ${ }^{\star}$ or subsitut ${ }^{\star}$ or treat $\left.{ }^{\star}\right)$ ).ab. or placebo*.ab,ti. or drug therapy.fs. or trial.ab,ti. or groups.ab. or (control ${ }^{\star}$ adj3 (trial ${ }^{\star}$ or study or studies)).ab,ti. or ((singl ${ }^{\star}$ or doubl ${ }^{\star}$ or tripl ${ }^{\star}$ or trebl $\left.{ }^{\star}\right)$ adj3 (blind ${ }^{\star}$ or mask or dummy $\left.^{\star}\right)$ ).mp. or clinical trial, phase ii/ or clinical trial, phase iii/ or clinical trial, phase iv/ or randomised controlled trial/ or pragmatic clinical trial/ or (quasi adj (experimental or random*)).ti,ab. or ((waitlist* or wait $^{\star}$ list $^{\star}$ or treatment as usual or TAU) adj3 (control or group)).ab.)

\section{4. (1 and 2 and 3 )}

Records were screened for reports of RCTs within the scope of the Cochrane Common Mental Disorders Group. Secondary reports of RCTs were tagged to the appropriate study record. The CCMD-CTR is current to June 2016 only.

Psychosocial interventions for survivors of rape and sexual assault experienced during adulthood (Protocol) 


\section{CONTRIBUTIONS OF AUTHORS}

Sarah Brown and Nazanin Khasteganan drafted the protocol, with regular discussion and input from Lorna O'Doherty. Katherine Brown, Kelsey Hegarty, Grace Carter, Laura Tarzia and Gene Feder reviewed the drafts.

$\mathrm{SB}$ is the guarantor for the review.

\section{DECLARATIONS OF INTEREST}

With the exception of Kelsey Hegarty and Laura Tarzia, all review authors are funded for their work on this review by the Multidisciplinary Evaluation of Sexual Assault Referral Centres for better Health (MESARCH) project; a project (number 16/117/04) funded by an institutional research grant from the National Institute for Health Research (NIHR) Health Service and Delivery Research Programme to Coventry University.

Sarah Brown (SB) - none known.

Nazanin Khasteganan (NK) - none known.

Katherine Brown (KB), in the interest of transparency, declares that she led a local evaluation of the Blue Sky Centre, a sexual assault referral centre in Warwickshire, between 2013 and 2015.

Kelsey Hegarty $(\mathrm{KH})$ declares institutional research funding from the National Health and Medical Research Council for a trial of screening and intervention in primary care. $\mathrm{KH}$ also declares monies paid to her from the World Health Organization to attend a guideline group on intimate partner violence, and funds from General Practice Victoria to deliver a training program on intimate partner violence for general practitioners.

Grace Carter (GC) - none known.

Laura Tarzia (LT) declares funding from the Australian Research Council to develop an online intervention for women experiencing intimate partner sexual violence, and funding from the University of Melbourne to develop a smartphone application for early intervention for students affected by sexual violence or dating violence.

Gene Feder (GF) declares that he is Chief Investigator or Co-investigator on a range of NIHR and MRC (Medical Research Council) grants. Lorna O'Doherty (LOD) declares that she is Chief Investigator on the MESARCH project.

\section{SOURCES OF SUPPORT}

\section{Internal sources}

- Coventry University, UK.

Funds 20\% of the MESARCH (Multidisciplinary Evaluation of Sexual Assault Referral Centres for better Health) project

\section{External sources}

- National Institute for Health Research Health Services and Delivery Research Programme, UK.

Funds $80 \%$ of the MESARCH project 